Int.J. Hum. Soc. Dev. Res.

ISSN (P):2521-1439; ISSN (E):2523-4331

Volume 4, Number 1, 2020. 41-73

DOI:10.30546/2523-4331.2020.4.1.41

\title{
THE EFFECT OF THE COMPANY PROFIT ON THE COMPANY AVERAGE LIFE IN OPERATIONS
}

\author{
Yunus KAYABAŞ \\ Near East University, Northern Cyprus
}

(C) The Author(s) 2020

\section{ABSTRACT}

The aim of this article is to reveal the opinions of the textile executives, who continue their activities in Bursa, on the effect of company profitability on the average life span of the company. In addition to the primary purpose of the research, there is also the question of whether there are significant differences in the effect of company profitability on the company's average life span according to demographic variables such as gender, age, marital status, educational status, years of work in the company, type of company, number of employees in the company, This study, prepared in line with the stated objectives, it is important to point out how management managers perceive the impact of the company's profitability, which is important for business management, on the life expectancy of the company to be sophisticated at the point of driving a drive to drive profitability and the life expectancy of the company. The universe of this research is the textile business managers in the field of activity in Bursa. Sampling was chosen randomly ( $\mathrm{n}=100)$. In the analysis of the data, a significance level of 0.05 was considered and all the analyzes explained were interpreted as appropriate for the purpose. Several hypotheses test results were examined by one-way ANOVA test and independent sample t-test. Regression analysis was applied to measure frequency distribution for all sizes, the effect of company profitability over the company life expectancy. As a result, it is concluded that the relationship between company profitability and company life expectancy is $\mathrm{p}<0.00$ significant, and company profitability has been found to make a meaningful contribution to company life expectancy.

C) 2020.All rights reserved

\section{ARTICLE HISTORY}

Received: 27/12/2019

Accepted: 23/02/2020

Published online: 27/04/2020

\section{KEYWORDS}

Company Profitability, Company Average Lifetime, Business, Textile,test

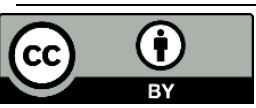

www.ijhsdr.com 


\section{Introduction}

Depending on the momentum in the historical development of man, the company's profitability and company life expectancy needs and expectations are changing rapidly. These rapidly changing needs and expectations of businesses have given a different dimension to business management. This dimension required a review of the company's profitability and the company's average life cycle. The profitability of the company and the life expectancy of the company are the result of the relationship between the features of the business and their expectations. The company profitability and the life expectancy of the company as a result of this relationship also shape the share of the operator in the market. To understand the company's profitability and the average life span of the company, you first need to examine the company's profitability and its role on the business of the company's average life span.

As Judith Williamson puts it, "The meaning that many businesses consciously choose in their lives consists of their expenses rather than their income." In the world, the prospect of increasing company profitability and average life expectancy of the company through public communication tools and modern advertising is being spurred, created and narrated As the number of businesses increasingly recognizes the possibilities offered to them, the number of enterprises that constitute their purpose of identity and life through "company profitability and life expectancy of the company" is increasing.

A business that wants to increase its share in the market should be able to control the company's profitability and company life expectancy variables. For this purpose, many companies have built a model of profitability and average life span of the company. The aim is to identify the key factors of the company's profitability and company life expectancy, and to determine other factors depending on these factors. However, studies have shown that there are those who can not be controlled, as long as they are variables that can be controlled within the company's profitability and company life expectancy.

Amortization in businesses; a prepayment of a paid advance payment, in accordance with years, to the business activities. When businesses distinguish between depreciation and intangible asset values, the losses that occur over time are transferred to cost calculations to keep the value of the operating capital under protection (Çakır, 2000 p.50).

Assets standing in the business are used directly during the production phases. Fixed assets are the most necessary factors of production stages such as raw materials and materials.

The production process adds all production factors such as raw materials and materials to the cost of a finished product. The use of fixed assets, a production factor, and the depreciation state must be added to the cost of the product. These additions are made by depreciation (Kirac1, 2000 p. 39).

While the sales price of a product produced in the enterprises is calculated; the cost of the depreciation is added to the profit of the operator. The worn out items in these assets are also added to the financials. When products are sold, the sale price, all cost 
factors and depreciation are also returned to the business accounts in cash (Aksoy, 1993, p. 33).

If accepted in the framework of the accrual basis, depreciation is shown as expense in the operating accounts. In other words, it is a kind of expense that only shows up on the accounting records that do not have the obligation to cash out. It is possible to mention cash as well as the amortization amount shown in the business records. This also means that a source of funding that is not cost-effective in businesses (Aygün, 2012,p.219).

Business spending they are willing to recapture often. For this reason, they want to pay back the fixed amount they received with advance payment, even if they have spread to these years. For this reason, they add depreciation to cost elements such as raw materials, materials, workmanship. In this way, the amount of profit is added to the cost items in which the depreciations are included and the sales proceeds are taken into consideration, and in the content of the profit to be obtained from these costs, the cash input is obtained by the name of the depreciation provisions. This cash work, which is profitable for business in a way too much, can be used to finance its capital (Çakır, 2000 p.50).

The most important goals (Clegg and Dunkerley, 2013,p. 4) for explaining the different types of business are explained by the most widely accepted ones; maintaining profitability, profitability, growth and prosperity (Barnard, 1938,p. 2, Rice, 1963,p. 4, Katz and Kahn, 1966,p. 6, Adizes, 1989,p. 3, Suárez and Utterback, 1995,p.415, De Geus, 1999,p. 3).

The literature evaluates the realization of the objectives of the enterprises within the concept of "performance". However, as Meyer and Zucker (1989) point out, there is no sufficient condition to ensure the continuity of high-level performance opera- tions. As long as the business continues, it will be possible to gain access to other objectives. Surveys of life spans of businesses (De Geus, 1999,p.8, ATO, 2005, Capital Online Monthly January 2010, 4) reveal that there is little evidence of business long life in support of Meyer and Zucker.

\section{Company Profitability}

Along with the difficulties of today's competitive conditions, businesses need to take some measures to maintain their continuity. These measures can be carried out with the appropriate amount of working capital in their hands. For this, they need to efficiently manage their working capital in their hands. The working capital represents the assets that the operator owns, that is, the assets that can be converted into cash within one year, or which are easily transferable, and which are included in the group of returning assets (Brigham, Houston, 2007, p. 63). Assets included in working capital are cash and cash-like assets (ready-to-value), securities, receivables and inventories. Investments in the operating capital are important for the enterprises to continue their operations in an uninterrupted manner and to continue their production effectively, to minimize the risks they can face and to keep their operations profitable. 


\section{Company Average Life Expectancy}

A request to put forward a plan to survive for a long time shows itself in Akin's thoughts. In this case, knowledge and theories are produced with traditional Western knowledge questions. Why are certain organizations alive too much from others? How do those who live for a long time achieve this? What are the reasons for not succeeding? Behind these questions, there is the assumption that whatever is in place is important and respected. These explanations. Hannan and Freeman (1977: 1984) have similarities to the means of validity of their organizations with their time of existence. What Akin (2000) attaches importance to the question of how the story is told, rather than the reality of what is long-lived. Because, if objects are not social constructs, continuity is a social construct. Just because of this, the issue of continuing to exist is related to psychology, religion, science and fiction.

There are some compulsory questions to be asked in terms of organization: When we ask for a long life review, what is the organization itself or the core identity? What is continuity or loss of life? For organizations that are not "live" objects, these are difficult questions to answer. But it is also the one that gives them their living qualities. Because objectified metaphors are used to give meaning to events / events, stories are adapted to revive them (Akin, 2000).

In this essay, as Kendall and Kendall (1993) point out, it is not random that one of the metaphors commonly used in defining the organization is an organism. How then should an organizational long life be defined? According to Akin (2000), the duration of the existence of an organization is sufficient for this definition. Because the duration of the organization's lifetime exposes the time it takes to legally close, and it includes clear processes such as organizational restructuring, development and organizational closure. However, there is no agreement on how the merger and acquisition process and the organizational structure of life can be handled. The same situation also raises an uncertainty about when the organization starts to work. According to Akin (2000), mergers, name changes, industry changes, new ownership, cause of confusion in determining the start date.

\section{Method}

In the method of research; After discussing the problem situation, purpose and significance that it supports, it focuses on the hypothesis, the universe and sampling and assumptions, the collection and analysis of data by considering the limitations.

\section{Problem}

Today, textile management managers; in order to keep up with the rapid developments experienced in economic, social, political and technological fields and to be able to keep up with the changes and maintain their assets, the profitability of the company should continue to have a positive effect on the average life of the company. The fact that businesses offer services in an environment where competition is high requires that the profitability of their businesses has a positive effect on the average life of the company in order to be different from its competitors. Not to be adversely affected by the complex structure of businesses, changing environment, rapidly increasing costs and developing technology; it is even considered to be a well-known 
and used concept among the company's profitability and the company's average lifetime managers to convert them into advantages. It is argued that the environment in which the service is provided in the textile sector as well as the interaction between the service provider and the service provider and the interaction between the customers receiving the same service have a significant effect on the consumption, satisfaction, satisfaction, intention of re-purchasing and trust in the business (Chaudhuri and Holbrook, 2001, p. 81, Karatepe and Avci, 2002,p.19). It is the main problem of this article to show the effect of the profitability of the company on the average life span of the company.

\section{The Purpose and Importance of Research}

The primary aim of this research is to show the opinions of the managers of the textile firms on the effect of the profitability of the textile companies on the average life span of the company. This study, prepared in line with the stated objectives, it is important that the profitability of the company which is important for the management of the enterprise has an effect on the life expectancy of the company and it is the quality that will shed light on the point of turning the business managers on the company profitability and the effect of the average life span.

\section{Universe and Sampling}

The universe of this research consists of the managers of textile firms operating in Bursa. The sample was selected randomly $(n=100)$.

\section{Model of research}



Figure 1. Model of the study

This research was conducted with descriptive research model. Descriptive research is a research model that attempts to explain the contexts, past or present events, concepts, and relationships related to a problem.

\section{Limitations of the Study}

- The research is limited to the dates of 01-30.01.2018 in the textile companies which are continuing their activities in the province of Bursa. 
- Allowing partial exploration in businesses can reduce the universe of researchers.

- Some administrators working in the business are given free leave because of the permission, some of the collection of data at the planned date range, such as illness and birth. it is impossible to reach because of such situations, in which case the research minimized the universe.

- Variables discussed in the survey are limited by the reliability dimension of the questionnaire applied.

- The fact that some of the administrators in the textile firms, which are continuing their activities in the province of Bursa, did not want to participate in the survey study also caused the sample of the research universities to shrink.

\section{Collection of Data}

Survey technique was used as data collection tool. In this survey application, managers were asked to fill in the survey questionnaires by face to face and fill out the questions in the questionnaire by telling them that they will be used only in a scientific work without any effect. Surveys were conducted in January 2018 for the managers of the identified textile companies.

In order to prepare the questionnaire which is a data collection tool, the literature on the company profitability and the average life span of the company was searched. In addition, researches related to the subject and questionnaires used in these researches have been examined. As a result of the work done, the survey was developed by the researcher.

While this form was being created, the information needed about the managers in the textile companies which are continuing their activities in the province of Bursa and the previous work done were taken into consideration.

The information form contains questions about socio-demographic information on gender, age, marital status, educational status, working time in the company, type of company, number of employees in the company, and competition status in the environment in which the business is located. As the data collection tools in the survey, a "personal information form" consisting of 8 questions prepared by the researcher in the direction of the literature was used and scales consisting of 61 items measuring the "company profitability" and "company life expectancy" applications were used.

\section{Company Profitability Scale}

Beykent University, Institute of Social Sciences, Department of Business Administration, Yildiz, who was written by Y1ldız Öztürk in 2015, Assoc. Dr. Erkut Altındağ has consulted on the "Modern Strategic Management Techniques Chaos Theory and the impact of the Blue Ocean Strategy on the company's performance" was taken from the master's thesis.

Company Average Life Scale

Professor Gül Başer from Akdeniz University Social Sciences Institute, Department of Business Administration, Assoc. Dr. An excerpt from the master's thesis titled "A Qualitative Research on Family Businesses with Continuity Challenge: Western Mediterranean Family Businesses" consulted by Ferda Erdem. 


\section{Research Hypotheses}

Quantitative research has been applied in this article study. The quantitative approach was formed at the beginning of the 20th century when the social sciences began to take shape and the research methods that science was using and the data collection techniques applied to the social sciences. The quantitative research model is a research model that can be observed, measured, and quantifiable by objectifying phenomena and events. In our research, a descriptive research model which is included in the quantitative research model has been applied.

The basic hypothesis of this research which aims to reveal the effect of the profitability of the companies of the textile firms which continue their activities in the province of Bursa to the life expectancy of the company is given below.

The basic hypothesis: The profitability of the company has an effect on the average life of the company.

\section{Analysis of Statistics Used in Research}

Statistical analyzes of the data obtained from the survey results were made using SPSS 23 package program and Windows computer program while analyzing the collected data in the study. Firstly demographic variables are grouped. In the analysis of the data, a significance level of 0.05 was considered and all the analyzes explained were interpreted as appropriate for the purpose. When the data were analyzed, Reliability Analysis was performed for all scaled questions and for Basic Dimensions. One-way ANOVA test and independent sample t-test results and various hypothesis test results were examined. Cronbach Alpha $(\alpha)$ test statistic was applied for the dimensions. Regression analysis was applied to measure the frequency distribution for all dimensions, the effect of company profitability on the company average life span.

\section{Results}

Socio-demographic findings

Table 1. Frequency Analysis of Demographical Situations of Managers

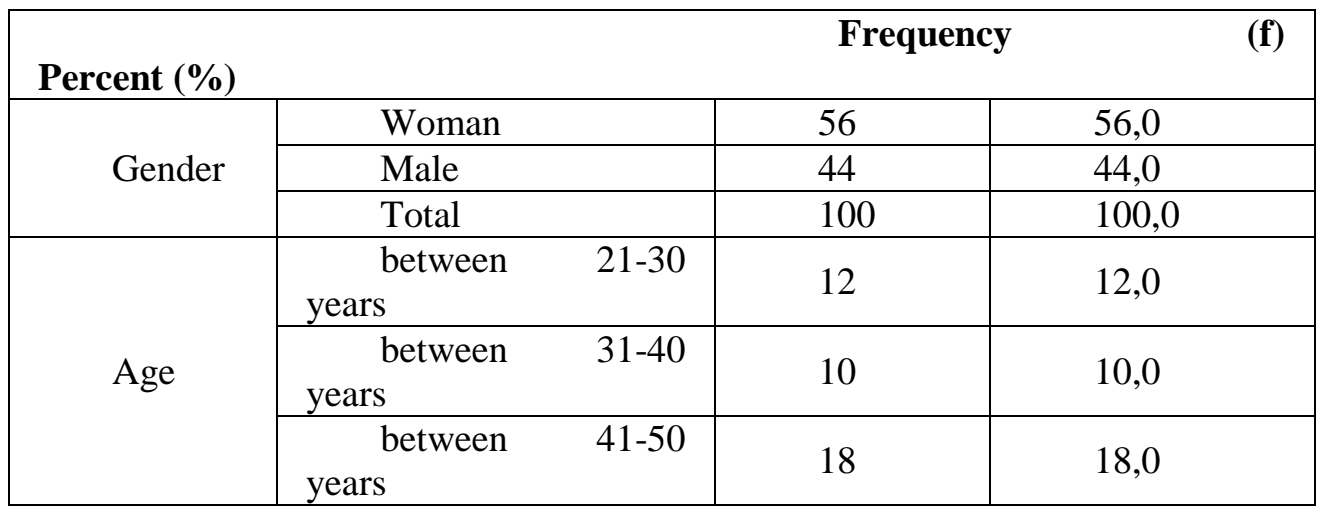




\begin{tabular}{|c|c|c|c|}
\hline & $\begin{array}{l}\text { between } 51-60 \\
\text { years }\end{array}$ & 31 & 31,0 \\
\hline & 60 years and over & 29 & 29,0 \\
\hline & Toplam & 100 & 100,0 \\
\hline \multirow{5}{*}{$\begin{array}{l}\text { Marital } \\
\text { status }\end{array}$} & Married & 18 & 18,0 \\
\hline & Single & 47 & 47,0 \\
\hline & Divorced & 11 & 11,0 \\
\hline & widowed & 24 & 24,0 \\
\hline & Total & 100 & 100,0 \\
\hline \multirow{7}{*}{$\begin{array}{l}\text { Educati } \\
\text { onal status }\end{array}$} & Primary school & 41 & 41,0 \\
\hline & Middle School & 16 & 16,0 \\
\hline & High school & 19 & 19,0 \\
\hline & Associate & 7 & 7,0 \\
\hline & $\begin{array}{l}\text { Bachelor } \\
\text { University }\end{array}$ & 8 & 8,0 \\
\hline & Graduate & 9 & 9,0 \\
\hline & Total & 100 & 100,0 \\
\hline \multirow{4}{*}{\begin{tabular}{l}
\multicolumn{2}{c}{ Workin } \\
$\mathrm{g}$ time in \\
company
\end{tabular}} & $1-5$ year & 60 & 60,0 \\
\hline & 6-10 year & 38 & 38,0 \\
\hline & 21 year and over & 2 & 2,0 \\
\hline & Total & 100 & 100,0 \\
\hline \multirow{4}{*}{$\begin{array}{l}\text { Compan } \\
\text { y type }\end{array}$} & $\begin{array}{cc}\text { Joint } & \text { Stock } \\
\text { Company (Inc) } & \end{array}$ & 23 & 23,0 \\
\hline & $\begin{array}{l}\text { Limited company } \\
\text { (Co. Ltd.) }\end{array}$ & 75 & 75,0 \\
\hline & $\begin{array}{l}\text { Cooperative } \\
\text { company }\end{array}$ & 2 & 2,0 \\
\hline & Total & 100 & 100,0 \\
\hline
\end{tabular}

According to the genders of the administrators participating in the survey; women $(\mathrm{n}=56)(56.0 \%)$, male $(\mathrm{n}=44)(44.0 \%)$, according to their age; between the ages of $21-30(n=12)(12.0 \%)$, between the ages of $31-40(n=10)(10,0 \%)$, between the ages of 41-50 $(n=18)(18.0 \%)$, between the ages of 51 and $60(n=31)(31.0 \%), 60$ years and over $(\mathrm{n}=29)(29.0 \%)$, according to marital status; married $(\mathrm{n}=18)(18.0 \%)$, single $(n=47)(47.0 \%)$, divorced $(n=11)(11.0 \%)$, His wife died $(n=24)(24.0 \%)$, according to their educational status; primary school $(\mathrm{n}=41)(41.0 \%)$, middle school $(\mathrm{n}=16)(16,0 \%)$, high school $(\mathrm{n}=19)(19,0 \%)$, associate degree $(\mathrm{n}=7)(7 \%)$, bachelor / university $(\mathrm{n}=8)(8 \%)$, post graduate $(\mathrm{n}=9)(9.0 \%)$, according to the working time in the company; between $1-5$ years $(n=60)(60.0 \%), 6$ to 10 years $(n=$ $38)(38.0 \%), 21$ years and over $(n=2)(2.0 \%)$, according to the company's product; Joint-stock company $(\mathrm{n}=23)(23,0 \%)$, limited company $(\mathrm{n}=75)(75,0 \%)$, The cooperative company $(n=2)(2.0 \%)$, according to the number of employees in the company; 0 to $9(\mathrm{n}=32)(32.0 \%), 10$ to $49(\mathrm{n}=68)(68.0 \%)$, according to the competition situation in the environment in which the operator is located; there was no competition $(n=14)(14,0 \%)$, there is some competition $(n=86)(86.0 \%)$. 
Table 2. Frequency Analysis of Demographical Situations of Managers (Continued)

\begin{tabular}{|l|l|c|c|}
\hline \multicolumn{2}{|l|}{} & Frequency & Percent \\
(\%)
\end{tabular}

According to the number of employees in the companies in which the managers participating in the survey are; 0 to $9(\mathrm{n}=32)(32.0 \%), 10$ to $49(\mathrm{n}=68)(68.0 \%)$, according to the competition situation of the companies in which they are located; with no competition $(n=14)(14.0 \%)$, with some competition $(n=86)(86.0 \%)$.

\section{Reliability Analysis Results for Basic Dimensions}

A reliability analysis was conducted for the survey questions used in the research. Cronbach's alpha test statistic was used for the reliability of the questionnaire. Evaluation criterion used in the evaluation of the Cronbach's alpha coefficient; If $0,00 \leq \alpha$ $<0,40$ the scale is not reliable. If $0,40 \leq \alpha<0,60$ the scale is low reliability. If $0,60 \leq \alpha$ $<0,80$, the scale is highly reliable. If $0,80 \leq \alpha<1,00$, the scale is evaluated as highly reliable. Questions about each scale and Cronbach Alpha values can be said to be at acceptable levels for the social sciences.

\section{Business Profitability Scale T-Test and Variance Analysis}

Table 3. Gender and Business Profitability Scale Group Analysis Table

\begin{tabular}{|c|c|c|c|c|c|}
\hline \multicolumn{6}{|c|}{ Group Analysis } \\
\hline & $\begin{array}{ll} & \text { Gende } \\
\mathrm{r} & \end{array}$ & & $\begin{array}{ll} & \text { Mea } \\
\mathrm{n} & \end{array}$ & $\begin{array}{c}\text { Std. } \\
\text { Deflection }\end{array}$ & $\begin{array}{l}\text { Std. Error } \\
\text { Mean }\end{array}$ \\
\hline \multirow[t]{2}{*}{$\begin{array}{c}\text { Business } \\
\text { Profitability }\end{array}$} & Woman & $5^{5}$ & 1,7128 & , 15791 & ,02111 \\
\hline & Male & $5^{4}$ & 1,7034 & , 16812 & ,02535 \\
\hline
\end{tabular}

As can be understood from the above table, while the operating profitability of 56 female managers participating in the research is the information averaging $(1,7128)$, the operational profitability of 44 male managers who have contributed to the study is 
determined as the information averaging (1,7034). Female managers have higher business profitability knowledge levels.

Table 4. Gender and Business Profitability Scale Group Analysis Independent Sample T-Test

\begin{tabular}{|c|c|c|c|c|c|c|c|c|c|c|}
\hline \multicolumn{11}{|c|}{ Independent Sample T-Test } \\
\hline & & \multicolumn{2}{|c|}{$\begin{array}{l}\text { Levene } \\
\text { Test }\end{array}$} & \multicolumn{7}{|c|}{ Equality of t-test averages } \\
\hline & & \multirow[t]{2}{*}{$\mathrm{F}$} & \multirow[t]{2}{*}{$\begin{array}{l}\text { S } \\
\text { ig. }\end{array}$} & \multirow[t]{2}{*}{$\mathrm{t}$} & \multirow[t]{2}{*}{ Df } & \multirow[t]{2}{*}{$\begin{array}{l}\text { Si } \\
\text { g.. } \\
(2- \\
\text { taile } \\
\text { d) }\end{array}$} & \multirow[t]{2}{*}{$\begin{array}{l}\text { Mean } \\
\text { Differe } \\
\text { nce }\end{array}$} & \multirow[t]{2}{*}{\begin{tabular}{l}
\multicolumn{1}{c}{ Std. } \\
Error \\
Differe \\
nce
\end{tabular}} & \multicolumn{2}{|c|}{\begin{tabular}{l}
\multicolumn{1}{c}{$95 \%$} \\
Difference \\
and \\
Confidence \\
Interval
\end{tabular}} \\
\hline & & & & & & & & & $\begin{array}{r}\text { Lo } \\
\text { wer }\end{array}$ & $\begin{array}{c}\text { Up } \\
\text { per }\end{array}$ \\
\hline 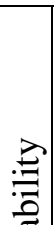 & $\begin{array}{l}\quad \text { Assu } \\
\text { ming } \\
\text { equal } \\
\text { varianc } \\
\text { e }\end{array}$ & $\begin{array}{l}21 \\
0\end{array}$ & $\begin{array}{l}65 \\
1\end{array}$ & $\begin{array}{l}29 \\
3\end{array}$ & 99 & $74^{, 7}$ & $54^{, 009}$ & $74^{, 032}$ & $\begin{array}{l}- \\
, 055 \\
43\end{array}$ & $\begin{array}{r}, 07 \\
449\end{array}$ \\
\hline 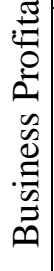 & \begin{tabular}{l}
\multicolumn{1}{c}{ Do } \\
not \\
assume \\
equal \\
varianc \\
e
\end{tabular} & & & $\begin{array}{l}29 \\
1\end{array}$ & $\begin{array}{r}89 \\
618\end{array}$ & ${ }_{75}^{, 7}$ & $\begin{array}{l}, 009 \\
54\end{array}$ & $9^{, 032}$ & $\begin{array}{l}- \\
, 056 \\
01\end{array}$ & $\begin{array}{c}, 07 \\
506\end{array}$ \\
\hline
\end{tabular}

Whether there is a meaningful difference between the profitability information of male and female managers is shown in the table above (2-tailed). When the value of significance is less than 0.05 , there is a meaningful difference between the two groups. The significance value of the analysis we have done is $0,774>0,05$, so it is understood that the information about the profitability of the male and female managers does not show any meaningful difference. $\mathrm{H}_{1}$ hypothesis is rejected because male and female managers' business profitability information has significance value $p>0.05$. In other words, the profitability information of men and women managers does not show any significant difference in terms of gender segmentation.

Table 5. Age and Business Profitability Descriptive Statistics Table

\begin{tabular}{|c|c|c|c|c|c|c|c|c|}
\hline \multicolumn{9}{|c|}{ Descriptive Statistics } \\
\hline \multicolumn{9}{|c|}{ Business Profitability } \\
\hline \multirow[t]{2}{*}{ Age } & \multirow[t]{2}{*}{$\mathrm{N}$} & \multirow[t]{2}{*}{ Mean } & \multirow[t]{2}{*}{$\begin{array}{l}\text { Std. } \\
\text { Deflectio } \\
\mathrm{n}\end{array}$} & \multirow[t]{2}{*}{$\begin{array}{r}\text { Std. } \\
\text { Error }\end{array}$} & \multicolumn{2}{|c|}{\begin{tabular}{l}
\multicolumn{1}{c}{ Mean } \\
confidence \\
interval
\end{tabular}} & \multirow[t]{2}{*}{$\begin{array}{l}\text { Mi } \\
\text { n. }\end{array}$} & \multirow[t]{2}{*}{$\begin{array}{l}\text { Ma } \\
\text { x. }\end{array}$} \\
\hline & & & & & $\begin{array}{r}\text { Lowe } \\
\text { r Limit }\end{array}$ & $\begin{array}{r}\text { Uppe } \\
\text { r Limit }\end{array}$ & & \\
\hline
\end{tabular}

Int.J. Hum. Soc. Dev. Res.

Volume 4, № 1, 2020.41-73 


\begin{tabular}{|c|c|c|c|c|c|c|c|c|}
\hline $\begin{array}{l}\text { betwe } \\
\text { en } 21- \\
30 \\
\text { years }\end{array}$ & $2^{1}$ & $35^{1,74}$ & ,09855 & $46^{, 028}$ & $\begin{array}{l}1,68 \\
08^{1,68}\end{array}$ & $\begin{array}{l}1,80 \\
60\end{array}$ & $\begin{array}{c}1, \\
58\end{array}$ & 89 \\
\hline \begin{tabular}{l}
\multicolumn{2}{c}{ betwe } \\
en $31-$ \\
40 \\
years
\end{tabular} & $0^{1}$ & $90^{1,72}$ &, 17722 & $05^{, 056}$ & $22^{1,60}$ & $\begin{array}{l}1,85 \\
57\end{array}$ & $\begin{array}{c}1, \\
55\end{array}$ & $\begin{array}{c}2, \\
16\end{array}$ \\
\hline $\begin{array}{l}\text { betwe } \\
\text { en } 41- \\
50 \\
\text { years }\end{array}$ & $8^{1}$ & $04^{1,70}$ & ,13901 & $77^{, 032}$ & $12^{1,63}$ & $94^{1,76}$ & 45 & 87 \\
\hline $\begin{array}{l}\text { betwe } \\
\text { en } 51- \\
60 \\
\text { years }\end{array}$ & $1^{3}$ & ${ }^{1,61}$ &, 18853 & $\begin{array}{l}, 033 \\
87\end{array}$ & $71^{1,54}$ & $\begin{array}{l}1,68 \\
54\end{array}$ & 42 & $\begin{array}{c}2, \\
05\end{array}$ \\
\hline \begin{tabular}{l}
\multicolumn{1}{c}{60} \\
years \\
and \\
over
\end{tabular} & $9^{2}$ & $14^{1,79}$ &, 10735 & $\begin{array}{l}, 019 \\
94^{,}\end{array}$ & $05^{1,75}$ & $\begin{array}{l}1,83 \\
21\end{array}$ & $\begin{array}{c}1, \\
61\end{array}$ & $\begin{array}{l}2, \\
00\end{array}$ \\
\hline $\begin{array}{l}\text { Topla } \\
\text { m }\end{array}$ & $\begin{array}{r}1 \\
00\end{array}$ & $\begin{array}{l}1,70 \\
88^{1,70}\end{array}$ & ,16172 & $\begin{array}{l}, 016 \\
18\end{array}$ & $\begin{array}{l}1,67 \\
66\end{array}$ & $\begin{array}{l}1,74 \\
08^{1,}\end{array}$ & $\begin{array}{l}1, \\
42\end{array}$ & $\begin{array}{c}2, \\
16\end{array}$ \\
\hline
\end{tabular}

The average profitability of managers aged 60 and over (1.7914) is the highest level among managers, while the average profitability of executives aged 51-60 years is the lowest $(1,6144)$.

Table 6. Age and Business Profitability Anova Table

\begin{tabular}{|c|c|c|c|c|c|c|}
\hline \multicolumn{7}{|l|}{ ANOVA } \\
\hline \multicolumn{7}{|c|}{ Business Profitability } \\
\hline & $\begin{array}{l}\text { Sum } \\
\text { of } \\
\text { squares }\end{array}$ & $\mathrm{f}^{\mathrm{d}}$ & $\begin{array}{l}\text { Squares } \\
\text { average }\end{array}$ & $\mathrm{F}$ & Sig & $\begin{array}{l}\text { Post-Hoc ve } \\
\text { Scheffe }\end{array}$ \\
\hline $\begin{array}{l}\text { Between } \\
\text { groups }\end{array}$ & ,483 & 4 &, 121 & $\begin{array}{l}5,4 \\
38\end{array}$ & $02^{, 0}$ & \multirow{3}{*}{$\begin{array}{l}51-60 \text { years, } 60 \\
\text { years and over> } \\
21-30 \text { years, } 31- \\
40 \text { years, } 41-50 \\
\text { years }\end{array}$} \\
\hline $\begin{array}{l}\text { Within } \\
\text { groups }\end{array}$ & 2,108 & $5^{9}$ & ,022 & & & \\
\hline Total & 2,590 & $9^{9}$ & & & & \\
\hline
\end{tabular}

According to $\mathrm{F}$ test result at $95 \%$ confidence level; The profitability of the managers' operating profitability was found to be $\mathrm{p}=0.002<0.05$. The $\mathrm{H}_{2}$ hypothesis is accepted when the manager's operational profitability is $\mathrm{p}<0.05$. As a result of the post-hoc and scheffe analysis on which group or groups the significance arises from; Between the ages of 51-60, the age group of 60 years and over participates higher than the other groups (between 21-30 years, between 31-40 years, between 41-50 years). 
Table 7. Descriptive Statistical Table for Marital Status and Business Profitability

\begin{tabular}{|c|c|c|c|c|c|c|c|c|}
\hline \multicolumn{9}{|c|}{ Descriptive Statistical } \\
\hline \multicolumn{9}{|c|}{ Business Profitability } \\
\hline \multirow[t]{2}{*}{$\begin{array}{l}\text { Marital } \\
\text { Status }\end{array}$} & \multirow[t]{2}{*}{$\mathrm{N}$} & \multirow[t]{2}{*}{ Mean } & \multirow[t]{2}{*}{$\begin{array}{c}\text { Std. } \\
\text { Deflectio } \\
n\end{array}$} & \multirow[t]{2}{*}{$\begin{array}{l}\text { Std. } \\
\text { Error }\end{array}$} & \multicolumn{2}{|c|}{$\begin{array}{l}\quad \text { Mean } \\
\text { confidence } \\
\text { interval }\end{array}$} & \multirow[t]{2}{*}{$\begin{array}{l}\text { Mi } \\
\text { n. }\end{array}$} & \multirow[t]{2}{*}{\begin{tabular}{|l} 
Ma \\
X.
\end{tabular}} \\
\hline & & & & & $\begin{array}{r}\text { Lowe } \\
r \text { Limit }\end{array}$ & $\begin{array}{r}\text { Uppe } \\
\text { r Limit }\end{array}$ & & \\
\hline $\begin{array}{c}\text { The } \\
\text { married }\end{array}$ & $8^{1}$ & $\begin{array}{l}1,81 \\
59\end{array}$ & ,07443 & $\begin{array}{l}{ }^{, 017} \\
54\end{array}$ & $\begin{array}{l}1,77 \\
88\end{array}$ & $2^{1,85}$ & $\begin{array}{c}1, \\
63\end{array}$ & $\begin{array}{c}1, \\
92\end{array}$ \\
\hline Single & $7^{4}$ & $\begin{array}{l}1,69 \\
27\end{array}$ & , 15618 & $\begin{array}{l}, 022 \\
78^{,}\end{array}$ & $\begin{array}{l}1,64 \\
68\end{array}$ & $8^{1,73}$ & $\begin{array}{l}1, \\
42\end{array}$ & $\begin{array}{c}2 \\
16\end{array}$ \\
\hline $\begin{array}{l}\text { Divorc } \\
\text { ed }\end{array}$ & ${ }^{1}$ & $\begin{array}{l}1,67 \\
95 \\
\end{array}$ &, 15155 & $\begin{array}{l}{ }^{, 045} \\
69 \\
\end{array}$ & $\begin{array}{l}1,57 \\
76 \\
\end{array}$ & $12^{1,78}$ & $\begin{array}{c}1, \\
53\end{array}$ & $\begin{array}{c}2, \\
00\end{array}$ \\
\hline \begin{tabular}{l}
\multicolumn{1}{c}{ His } \\
wife \\
passed \\
away
\end{tabular} & $4^{2}$ & $33^{1,67}$ & , 19615 & $04^{, 040}$ & $\begin{array}{l}1,59 \\
04^{1,59}\end{array}$ & $61^{1,75}$ & $\begin{array}{c}1, \\
45\end{array}$ & $\begin{array}{l}2 \\
05\end{array}$ \\
\hline Total & $\begin{array}{r}1 \\
00 \\
\end{array}$ & $\begin{array}{l}1,70 \\
88^{1,70}\end{array}$ & , 16171 & $\begin{array}{l}y^{, 016} \\
17\end{array}$ & $\begin{array}{l}1,67 \\
66\end{array}$ & $\begin{array}{l}1,74 \\
08^{1,}\end{array}$ & $\begin{array}{l}1, \\
42\end{array}$ & $\begin{array}{c}2, \\
16\end{array}$ \\
\hline
\end{tabular}

Marital status groups from managers are married with the highest average level of operating profitability $(1,8159)$, while marital status groups have the lowest average level of operating profitability of managers who have passed away $(1,6733)$.

Table 8. Profitability and Business Profitability Anova Table

\begin{tabular}{|c|c|c|c|c|c|c|}
\hline \multicolumn{7}{|c|}{ ANOVA } \\
\hline \multicolumn{7}{|c|}{ Business Profitability } \\
\hline & $\begin{array}{l}\text { Sum } \\
\text { of } \\
\text { squares }\end{array}$ & $\mathrm{f}^{\mathrm{d}}$ & $\begin{array}{l}\text { Squar } \\
\text { es } \\
\text { average }\end{array}$ & $\mathrm{F}$ & Sig & Post-Hoc ve Scheffe \\
\hline $\begin{array}{l}\text { Betwe } \\
\text { en } \\
\text { groups }\end{array}$ & ,259 & 3 &, 086 & $\begin{array}{l}3,5 \\
45\end{array}$ & $18^{, 0}$ & \multirow{3}{*}{$\begin{array}{l}\text { Single, Divorced> The } \\
\text { married, His wife passed } \\
\text { away }\end{array}$} \\
\hline $\begin{array}{l}\text { Within } \\
\text { groups }\end{array}$ & $2^{2,33}$ & $6^{9}$ &, 024 & & & \\
\hline Total & $0^{2,59}$ & $9^{9}$ & & & & \\
\hline
\end{tabular}

According to $\mathrm{F}$ test result at $95 \%$ confidence level; The profitability of the managers' profitability was found as $\mathrm{p}=0,018<0,05 . \mathrm{H}_{3}$ hypothesis is accepted when the profitability of managers' operational value is $p<0.05$. As a result of the post-hoc and scheffe analysis on which group or groups the significance arises from; Single, Married status of marriage group is higher than other groups (Married, Divorced). 
Table 9. Descriptive Statistical Table for Educational Status and Business Profitability

\begin{tabular}{|c|c|c|c|c|c|c|c|c|}
\hline \multicolumn{9}{|c|}{ Tanımlayıcı İstatistik } \\
\hline \multicolumn{9}{|c|}{ Business Profitability } \\
\hline \multirow[t]{2}{*}{$\begin{array}{l}\text { Educatio } \\
\text { nal Status }\end{array}$} & \multirow[t]{2}{*}{$\mathrm{N}$} & \multirow[t]{2}{*}{${ }_{n}^{\text {Mea }}$} & \multirow[t]{2}{*}{$\begin{array}{l}\text { Std. } \\
\text { Deflectio } \\
\mathrm{n}\end{array}$} & \multirow[t]{2}{*}{$\begin{array}{l}\text { Std. } \\
\text { Error }\end{array}$} & \multicolumn{2}{|c|}{$\begin{array}{l}\text { Mean } 95 \% \\
\text { confidence } \\
\text { interval }\end{array}$} & \multirow[t]{2}{*}{$\begin{array}{l}\text { Mi } \\
\text { n. }\end{array}$} & \multirow[t]{2}{*}{$\begin{array}{l}\mathrm{M} \\
\mathrm{ax} .\end{array}$} \\
\hline & & & & & $\begin{array}{l}\text { Low } \\
\text { er } \\
\text { Limit }\end{array}$ & $\begin{array}{r}\text { Uppe } \\
\text { r Limit }\end{array}$ & & \\
\hline $\begin{array}{l}\text { Primary } \\
\text { school }\end{array}$ & $1^{4}$ & $\begin{array}{l}1,65 \\
54\end{array}$ & , 18392 & $7^{, 028}$ & $\begin{array}{l}1,59 \\
73\end{array}$ & $\begin{array}{l}1,71 \\
34\end{array}$ & $\begin{array}{l}1, \\
42\end{array}$ & $\begin{array}{c}2, \\
16\end{array}$ \\
\hline $\begin{array}{l}\text { Middle } \\
\text { School }\end{array}$ & $6^{1}$ & $\begin{array}{l}1,72 \\
38\end{array}$ & , 12749 & $\begin{array}{l}, 031 \\
88^{, 031}\end{array}$ & $\begin{array}{l}1,65 \\
58\end{array}$ & $\begin{array}{l}1,79 \\
16\end{array}$ & $\begin{array}{r}1, \\
45\end{array}$ & $\begin{array}{l}1, \\
84\end{array}$ \\
\hline $\begin{array}{r}\text { High } \\
\text { school }\end{array}$ & $9^{1}$ & $\begin{array}{l}1,79 \\
23\end{array}$ & ,08501 & $\begin{array}{l}{ }^{, 019} \\
51\end{array}$ & $\begin{array}{l}1,75 \\
13\end{array}$ & $32^{1,83}$ & $\begin{array}{c}1, \\
61\end{array}$ & $\begin{array}{l}1, \\
95\end{array}$ \\
\hline $\mathrm{e}^{\text {Associat }}$ & 7 & $\begin{array}{l}1,81 \\
96\end{array}$ & , 10897 & $1^{, 041}$ & $\begin{array}{l}1,71 \\
88^{1,}\end{array}$ & $\begin{array}{l}1,92 \\
03\end{array}$ & $\begin{array}{c}1, \\
74\end{array}$ & $\begin{array}{c}2, \\
00\end{array}$ \\
\hline $\begin{array}{l}\text { Bachelor } \\
\text { / University }\end{array}$ & 8 & $\begin{array}{l}1,57 \\
90\end{array}$ &, 16585 & $6^{, 058}$ & $03^{1,44}$ & $7^{1,71}$ & $\begin{array}{c}1, \\
47\end{array}$ & $\begin{array}{l}1, \\
95\end{array}$ \\
\hline Master & 9 & $\begin{array}{l}1,77 \\
79\end{array}$ & , 10117 & $73^{, 033}$ & $\begin{array}{l}1,70 \\
00\end{array}$ & $\begin{array}{l}1,85 \\
55\end{array}$ & $\begin{array}{l}1, \\
63\end{array}$ & $\begin{array}{c}1, \\
92\end{array}$ \\
\hline Total & $\begin{array}{r}1 \\
00 \\
\end{array}$ & $8^{1,70}$ & , 16172 & ${ }^{, 016}$ & $\begin{array}{l}1,67 \\
66\end{array}$ & $\begin{array}{l}1,74 \\
08\end{array}$ & $\begin{array}{l}1, \\
42\end{array}$ & $\begin{array}{c}2, \\
16\end{array}$ \\
\hline
\end{tabular}

While the operating profitability average of the education groups of the managers is the highest $(1,8196)$, the operating profitability average of the managers who are the education groups, Bachelor / University is the lowest level (1,5790).

Table 10. Education and Business Profitability Anova Table

\begin{tabular}{|c|c|c|c|c|c|c|}
\hline \multicolumn{7}{|l|}{ ANOVA } \\
\hline \multicolumn{7}{|c|}{ Business Profitability } \\
\hline & $\begin{array}{l}\text { Sum of } \\
\text { squares }\end{array}$ & $\mathrm{f}^{\mathrm{d}}$ & $\begin{array}{l}\text { Squares } \\
\text { average }\end{array}$ & $\mathrm{F}$ & Sig & $\begin{array}{l}\text { Post-Hoc } \\
\text { Scheffe }\end{array}$ \\
\hline $\begin{array}{l}\text { Between } \\
\text { groups }\end{array}$ & ,517 & 5 &, 103 & $\begin{array}{l}4,6 \\
87\end{array}$ & $\begin{array}{l}0 \\
02\end{array}$ & \multirow{3}{*}{$\begin{array}{l}\text { Primary school, } \\
\text { High } \\
\text { Associate } \\
\text { Bachelor } \\
\text { University, } \\
\text { Master> Middle } \\
\text { School }\end{array}$} \\
\hline $\begin{array}{l}\text { Within } \\
\text { groups }\end{array}$ & 2,072 & $4^{9}$ & ,022 & & & \\
\hline Total & 2,589 & $9^{9}$ & & & & \\
\hline
\end{tabular}

According to $\mathrm{F}$ test result at $95 \%$ confidence level; The profitability of the managers' operating profitability was found to be $\mathrm{p}=0.002<0.05$. $\mathrm{H}_{4}$ hypothesis is accepted when 
the profitability of managers' operational value is $\mathrm{p}<0.05$. As a result of the post-hoc and scheffe analysis on which group or groups the significance arises from; Primary school, high school, associate degree, bachelor / university, graduate education group are higher than the other groups (secondary school).

Table 11. Working Time in Company and Descriptive Statistical Table for Operating Profitability

\begin{tabular}{|c|c|c|c|c|c|c|c|c|}
\hline \multicolumn{9}{|c|}{ Descriptive Statistical } \\
\hline \multicolumn{9}{|c|}{ Business Profitability } \\
\hline \multirow{3}{*}{$\begin{array}{l}\text { Workin } \\
\mathrm{g} \text { Time } \\
\text { in } \\
\text { Compan } \\
\text { y }\end{array}$} & \multirow[t]{3}{*}{$\mathrm{N}$} & \multirow[t]{3}{*}{ Mean } & \multirow{3}{*}{$\begin{array}{l}\text { Std. } \\
\text { Deflectio } \\
\mathrm{n}\end{array}$} & \multirow{3}{*}{$\begin{array}{l}\text { Std. } \\
\text { Error }\end{array}$} & \multirow{2}{*}{\multicolumn{2}{|c|}{\begin{tabular}{l}
\multicolumn{1}{c}{ Mean } \\
confidence \\
interval
\end{tabular}}} & \multirow{3}{*}{$\begin{array}{l}\text { Mi } \\
\text { n. }\end{array}$} & \multirow{3}{*}{$\begin{array}{l}\mathrm{Ma} \\
\mathrm{x} .\end{array}$} \\
\hline & & & & & & & & \\
\hline & & & & & $\begin{array}{r}\text { Lowe } \\
\text { r Limit }\end{array}$ & $\begin{array}{r}\text { Uppe } \\
\text { r Limit }\end{array}$ & & \\
\hline $\begin{array}{c}1-5 \\
\text { year }\end{array}$ & $0^{6}$ & $\begin{array}{l}1,74 \\
35\end{array}$ & ,13599 & $\begin{array}{l}5^{, 017} \\
56\end{array}$ & $\begin{array}{l}1,70 \\
83\end{array}$ & $\begin{array}{l}1,77 \\
85\end{array}$ & $\begin{array}{r}1, \\
45\end{array}$ & $\begin{array}{r}2, \\
05\end{array}$ \\
\hline $\begin{array}{c}\text { 6-10 } \\
\text { year }\end{array}$ & $8^{3}$ & $2^{1,65}$ & , 18771 & $46^{, 030}$ & $\begin{array}{l}1,59 \\
07\end{array}$ & $\begin{array}{l}1,71 \\
40\end{array}$ & $\begin{array}{r}1, \\
42\end{array}$ & $\begin{array}{c}2, \\
16\end{array}$ \\
\hline $\begin{array}{l}21 \\
\text { year } \\
\text { and } \\
\text { over }\end{array}$ & 2 & $\begin{array}{l}1,73 \\
69\end{array}$ & ,00000 & $\begin{array}{l}, 000 \\
00^{, 0}\end{array}$ & $68^{1,73}$ & $68^{1,73}$ & $\begin{array}{c}1, \\
74\end{array}$ & $\begin{array}{c}1, \\
74\end{array}$ \\
\hline Total & $\begin{array}{r}1 \\
00\end{array}$ & $\begin{array}{l}1,70 \\
88\end{array}$ &, 16172 & $\begin{array}{l}{ }^{, 016} \\
18\end{array}$ & $\begin{array}{l}1,67 \\
66\end{array}$ & $\begin{array}{l}1,74 \\
08\end{array}$ & $\begin{array}{c}1, \\
42\end{array}$ & $\begin{array}{c}2, \\
16\end{array}$ \\
\hline
\end{tabular}

While the operating profit group average of the managers in the company is between 1 and 5 years at the highest level (1.7435), the operating profitability average of managers with 6-10 years in the working hours group at the company is the lowest level $(1,6525)$.

Table 12. Working Time in the Company and Operating Profitability Anova Table

\begin{tabular}{|c|c|c|c|c|c|c|}
\hline \multicolumn{7}{|l|}{ ANOVA } \\
\hline \multicolumn{7}{|c|}{ Business Profitability } \\
\hline & $\begin{array}{l}\text { Sum of } \\
\text { squares }\end{array}$ & $\mathrm{f}^{\mathrm{d}}$ & $\begin{array}{l}\text { Squares } \\
\text { average }\end{array}$ & $\mathrm{F}$ & Sig & $\begin{array}{l}\text { Post-Hoc } \\
\text { Scheffe }\end{array}$ \\
\hline $\begin{array}{l}\text { Between } \\
\text { groups }\end{array}$ & , 196 & 2 & ,097 & $\begin{array}{l}3,9 \\
41\end{array}$ & $24^{, 0}$ & \multirow{3}{*}{$\begin{array}{l}1-5 \text { year> 6-10 } \\
\text { year, } \\
21 \text { year and over }\end{array}$} \\
\hline $\begin{array}{l}\text { Within } \\
\text { groups }\end{array}$ & 2,395 & $7^{9}$ &, 025 & & & \\
\hline Total & 2,590 & $9^{9}$ & & & & \\
\hline
\end{tabular}

According to $\mathrm{F}$ test result at $95 \%$ confidence level; The profitability of managers' business sense was found to be $\mathrm{p}=0,024<0,05 . \mathrm{H}_{5}$ hypothesis is accepted when the profitability of managers' operational value is $\mathrm{p}<0,05$. 
Table 13. Descriptive Statistical Table of Company Type and Operational Profitability

\begin{tabular}{|c|c|c|c|c|c|c|c|c|}
\hline \multicolumn{9}{|c|}{ Descriptive Statistical } \\
\hline \multicolumn{9}{|c|}{ Business Profitability } \\
\hline \multirow[t]{2}{*}{$\begin{array}{l}\text { Company } \\
\text { Type }\end{array}$} & \multirow[t]{2}{*}{$\mathrm{N}$} & \multirow[t]{2}{*}{ Mea } & \multirow[t]{2}{*}{$\begin{array}{l}\text { Std. } \\
\text { Deflectio } \\
n\end{array}$} & \multirow[t]{2}{*}{$\begin{array}{l}\text { Std. } \\
\text { Error }\end{array}$} & \multicolumn{2}{|c|}{$\begin{array}{l}\text { Mean } 95 \% \\
\text { confidence } \\
\text { interval }\end{array}$} & \multirow[t]{2}{*}{$\begin{array}{l}\mathrm{Mi} \\
\mathrm{n} .\end{array}$} & \multirow[t]{2}{*}{$\begin{array}{l}\mathrm{M} \\
\mathrm{ax} .\end{array}$} \\
\hline & & & & & $\begin{array}{l}\text { Low } \\
\text { er } \\
\text { Limit } \\
\end{array}$ & $\begin{array}{r}\text { Uppe } \\
\text { r Limit }\end{array}$ & & \\
\hline \begin{tabular}{l}
\multicolumn{1}{c}{ Joint } \\
Stock \\
Company \\
(Inc)
\end{tabular} & $3^{2}$ & $8^{1,64}$ & , 15399 & $11^{, 032}$ & $22^{1,58}$ & $53^{1,71}$ & 42 & $\begin{array}{c}1, \\
89\end{array}$ \\
\hline $\begin{array}{l}\text { Limited } \\
\text { company } \\
\text { (Co. Ltd.) }\end{array}$ & $5^{7}$ & $29^{1,72}$ & , 16103 & $5^{, 018}$ & $5^{1,68}$ & $\begin{array}{l}1,75 \\
99\end{array}$ & $\begin{array}{c}1, \\
45\end{array}$ & $\begin{array}{c}2, \\
16\end{array}$ \\
\hline $\begin{array}{l}\text { Cooperati } \\
\text { ve } \\
\text { company }\end{array}$ & 2 & $85^{1,86}$ & ,00000 & $00^{, 000}$ & $\begin{array}{l}1,86 \\
84^{1,86}\end{array}$ & $84^{1,86}$ & $\begin{array}{c}1 \\
87\end{array}$ & $\begin{array}{l}1, \\
87\end{array}$ \\
\hline Total & $\begin{array}{r}1 \\
00\end{array}$ & $\begin{array}{l}1,70 \\
86\end{array}$ & , 16172 & $\begin{array}{l}7^{, 016} \\
17^{-016}\end{array}$ & $\begin{array}{l}1,67 \\
66\end{array}$ & $\begin{array}{l}1,74 \\
08^{1,74}\end{array}$ & $\begin{array}{c}1 \\
42\end{array}$ & $\begin{array}{r}2, \\
16\end{array}$ \\
\hline
\end{tabular}

The operating profitability average of the company type groups in which the managers are working cooperatively is the highest level $(1,8685)$, while the average of the operating profitability of the managers who are the type of company type corporations (corporations) is the lowest $(1,6488)$.

Table 14. Type of Company and Profitability of Operation Anova Table

\begin{tabular}{|l|c|r|l|l|c|}
\hline \multicolumn{7}{|l|}{ ANOVA } \\
\hline Business Profitability & Sum of squares & df & Squares average & F & Sig. \\
\hline Between groups &, 150 & 2 &, 074 & 2,95 &, 05 \\
\hline Within groups & 2,441 & 9 &, 025 & & 8 \\
\hline Total & 2,590 & 9 & & & \\
\hline
\end{tabular}

According to $\mathrm{F}$ test result at $95 \%$ confidence level; The profitability of managers' operational value was found to be $\mathrm{p}=0,058>0,05 . \mathrm{H}_{6}$ hypothesis is rejected if the manager's profitability for business is $\mathrm{p}>0.05$.

Table 15. Number of Employees in the Company and Operating Profitability Scale Group Analysis Table 


\begin{tabular}{|c|c|c|c|c|cc|}
\hline \multicolumn{1}{|c|}{ Group Analysis } & $\begin{array}{l}\text { Number } \\
\text { Employees in the } \\
\text { Company }\end{array}$ & $\mathrm{N}$ & Mean & $\begin{array}{c}\text { Std. } \\
\text { Deflection }\end{array}$ & $\begin{array}{c}\text { Std. } \\
\text { Mean }\end{array}$ & Error \\
\cline { 2 - 7 } $\begin{array}{c}\text { Business } \\
\text { Profitability }\end{array}$ & $0-9$ between & $\begin{array}{c}3 \\
2\end{array}$ & 5 &, 12726 &, 02251 & \\
\cline { 2 - 7 } & $10-49$ between & $\begin{array}{c}6 \\
8\end{array}$ & 4 &, 16122 & \multirow{2}{*}{, 01956} & \\
\hline
\end{tabular}

As can be understood from the above table, while the average number of employees working in 32 companies participating in the survey was between 0 and 9 , the operating profitability average (1.7945) was found, while the 68 companies contributing to the survey were found to have an average operating profitability $(1,6684)$. Managers with 0 9 employees in the company have higher operating profitability levels.

Table 16. Number of Employees in the Company and Operating Profitability Scale Group Analysis Independent Sample T-Test

\begin{tabular}{|c|c|c|c|c|c|c|c|c|c|c|}
\hline \multicolumn{11}{|c|}{ Independent Sample T-Test } \\
\hline & & \multicolumn{2}{|c|}{$\begin{array}{l}\text { Levene } \\
\text { Test }\end{array}$} & \multicolumn{7}{|c|}{ Equality of t-test averages } \\
\hline & & \multirow[t]{2}{*}{$\mathrm{F}$} & \multirow[t]{2}{*}{$\begin{array}{l}\text { S } \\
\text { ig. }\end{array}$} & \multirow[t]{2}{*}{$\mathrm{t}$} & \multirow[t]{2}{*}{ Df } & \multirow[t]{2}{*}{$\begin{array}{l}\text { Si } \\
\text { g.. } \\
(2- \\
\text { taile } \\
\text { d) }\end{array}$} & \multirow[t]{2}{*}{$\begin{array}{l}\text { Mean } \\
\text { Differe } \\
\text { nce }\end{array}$} & \multirow[t]{2}{*}{\begin{tabular}{l}
\multicolumn{1}{c}{ Std. } \\
Error \\
Differe \\
nce
\end{tabular}} & \multicolumn{2}{|c|}{$\begin{array}{l}\text { 95\% } \\
\text { Difference } \\
\text { and } \\
\text { Confidence } \\
\text { Interval }\end{array}$} \\
\hline & & & & & & & & & Alt & Üst \\
\hline  & $\begin{array}{l}\text { Assu } \\
\text { ming } \\
\text { equal } \\
\text { varianc } \\
\text { e } \\
\end{array}$ & $\begin{array}{r}3, \\
246\end{array}$ & $\begin{array}{l}07 \\
5\end{array}$ & $\begin{array}{r}3, \\
887\end{array}$ & 98 & $00^{, 0}$ & $06^{, 126}$ & $43^{, 032}$ & $\begin{array}{l}, 0 \\
617 \\
0\end{array}$ & $\begin{array}{l}, 1 \\
904 \\
3\end{array}$ \\
\hline 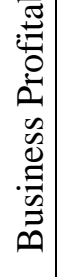 & \begin{tabular}{l}
\multicolumn{1}{c}{ Do } \\
not \\
assume \\
equal \\
varianc \\
e
\end{tabular} & & & $\begin{array}{r}4, \\
230\end{array}$ & $\begin{array}{r}75 \\
, 564\end{array}$ & $00^{, 0}$ & $06^{, 126}$ & $\begin{array}{l}, 029 \\
80\end{array}$ & $\begin{array}{l}, 0 \\
667 \\
0\end{array}$ & \begin{tabular}{l}
\multicolumn{1}{c}{1} \\
854 \\
3
\end{tabular} \\
\hline
\end{tabular}

According to the number of employees in the company, whether there is a meaningful difference between the profitability of the managers and the operating profit is shown in the table above. (2-tailed) we can understand the value of. When the value of significance is less than 0.05 , there is a meaningful difference between the two groups. Since the significance value of the analysis we conducted is $0,000<0.05$, it is understood that the operating profitability of managers is significantly different according to the number of employees in the company. According to the number of employees in the company, $\mathrm{H}_{7}$ hypothesis is accepted when the operating profitability 
of managers is $\mathrm{p}<0.05$. In other words, the operating profitability of managers differs significantly in terms of number of employees in the company.

Table 17. Group Competitiveness and Operability Profitability Scale Group Analysis Table

\begin{tabular}{|c|c|c|c|c|c|}
\hline \multicolumn{1}{|c|}{ Grup Analizi } \\
\hline \multirow{4}{*}{$\begin{array}{c}\text { Business } \\
\text { Profitability }\end{array}$} & $\begin{array}{l}\text { The Competitive Situation } \\
\text { of the Region in which the } \\
\text { Operator }\end{array}$ & N & Mean & $\begin{array}{c}\text { Std. } \\
\text { Deflection }\end{array}$ & $\begin{array}{c}\text { Std. } \\
\text { Error } \\
\text { Mean }\end{array}$ \\
\cline { 2 - 7 } & There is no competition & 1 & 1,64 &, 08683 &, 023 \\
\cline { 2 - 7 } & There is some competition & 8 & $\begin{array}{c}1,71 \\
9\end{array}$ &, 16890 & 22 \\
\hline
\end{tabular}

As can be understood from the above table, 14 operators who participated in the survey did not have any competition in the environment, and the expression of manager's profitability average $(1,6448)$ was determined as the average of the operating profitability of the manager $(1,7192)$ It was. Managers who say that there is some competition in the environment where the business is located have higher business profitability levels.

Table 18. The Survey of the Competitive State and Operability Profitability of the Region in which the Business Operated Group Analysis Independent Sampling T-Test

\begin{tabular}{|c|c|c|c|c|c|c|c|c|c|c|}
\hline \multicolumn{11}{|c|}{ Independent Sample T-Test } \\
\hline & & \multicolumn{2}{|c|}{$\begin{array}{l}\text { Levene } \\
\text { Test }\end{array}$} & \multicolumn{7}{|c|}{ Equality of t-test averages } \\
\hline & & \multirow[t]{2}{*}{$\mathrm{F}$} & \multirow[t]{2}{*}{$\begin{array}{l}\text { S } \\
\text { ig. }\end{array}$} & \multirow[t]{2}{*}{$\mathrm{t}$} & \multirow[t]{2}{*}{ Df } & \multirow[t]{2}{*}{$\begin{array}{l}\text { Si } \\
\text { g. } \\
(2- \\
\text { taile } \\
\text { d) }\end{array}$} & \multirow[t]{2}{*}{$\begin{array}{l}\text { Mean } \\
\text { Differe } \\
\text { nce }\end{array}$} & \multirow[t]{2}{*}{\begin{tabular}{l}
\multicolumn{1}{c}{ Std. } \\
Error \\
Differe \\
nce
\end{tabular}} & \multicolumn{2}{|c|}{$\begin{array}{l}\quad 95 \% \\
\text { Difference } \\
\text { and } \\
\text { Confidence } \\
\text { Interval }\end{array}$} \\
\hline & & & & & & & & & $\begin{array}{c}\text { Lo } \\
\text { wer }\end{array}$ & $\begin{array}{l}\text { Up } \\
\text { per }\end{array}$ \\
\hline 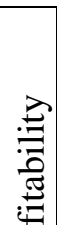 & $\begin{array}{l}\text { Assu } \\
\text { ming } \\
\text { equal } \\
\text { varianc } \\
\text { e }\end{array}$ & $\begin{array}{r}6, \\
440\end{array}$ & $\begin{array}{l}01 \\
4\end{array}$ & $\begin{array}{l}- \\
1,6 \\
09\end{array}$ & 99 & $12^{, 1}$ & , 07437 & $25^{, 046}$ & $\begin{array}{l}- \\
, 166 \\
12\end{array}$ & $\begin{array}{l}, 0 \\
174 \\
1\end{array}$ \\
\hline $\begin{array}{c}0 \\
0 \\
0 \\
w \\
0 \\
0 \\
0 \\
0 \\
0 \\
0\end{array}$ & \begin{tabular}{l}
\multicolumn{1}{c}{ Do } \\
not \\
assume \\
equal \\
varianc
\end{tabular} & & & $\begin{array}{l}- \\
2,5 \\
22\end{array}$ & $\begin{array}{r}32 \\
, 088\end{array}$ & $18^{, 0}$ & , 07437 & 51 & $\begin{array}{l}- \\
, 134 \\
44\end{array}$ & $\begin{array}{l}- \\
, 014 \\
21\end{array}$ \\
\hline
\end{tabular}




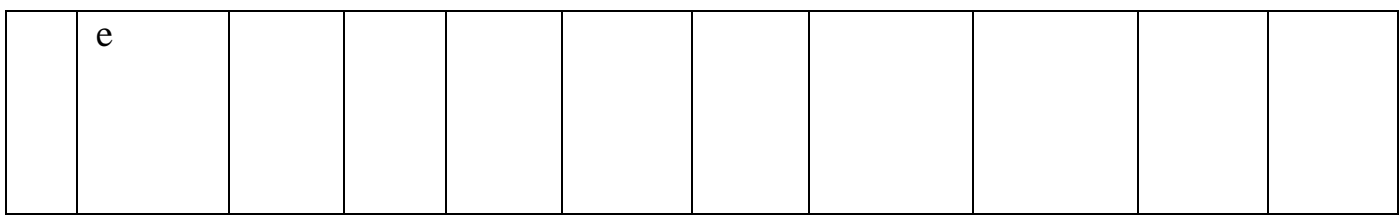

Whether there is a meaningful difference between the operating profitability of the managers according to whether or not there is competition in the environment in which the business is located is shown in the table above. (2-tailed) we can understand the value of. When the value of significance is less than 0.05 , there is a meaningful difference between the two groups. Since we have a significance level of 0,112>0,05 in our analysis, it is understood that the operational profitability of the managers does not show any meaningful difference according to whether or not there is competition in the environment. $\mathrm{H}_{8}$ hypothesis is rejected because the significance value of the managers' profitability is $p>0,05$ according to whether or not there is competition in the environment where the business is located. In other words, the operating profitability of managers does not differ significantly in terms of partitioning the situation of competition in the environment in which the operator is located.

\section{Company Average Lifetime Scale Reliability Analysis}

Table 19. Reliability Analysis of the Company Average Lifetime Scale

\begin{tabular}{|l|l|}
\hline \multicolumn{2}{|l|}{ Reliability Analysis } \\
\hline Cronbach's Alpha & N of Items \\
\hline, 696 & 23 \\
\hline
\end{tabular}

The internal consistency coefficient of the company's life expectancy scale was calculated. As a result of the analysis, Cronbach's alpha value was determined as 0,696.

Table 20. Reliability Analysis Results for the Company Average Life Survey

\begin{tabular}{|l|l|}
\hline $\begin{array}{l}\text { From time to time rumor spreads to the business to make } \\
\text { something happen. }\end{array}$ & Cronbach's \\
\hline $\begin{array}{l}\text { Certain promises (such as a chef, foreman, etc.) are available when } \\
\text { they adopt their views. }\end{array}$ &, 733 \\
\hline $\begin{array}{l}\text { Employees who adopt their own views are expected to provide a } \\
\text { well-equipped workspace. }\end{array}$ &, 703 \\
\hline \begin{tabular}{l} 
It acts politically (as if showing power), embracing his views. \\
\hline $\begin{array}{l}\text { It gives the employees deliberately different information and } \\
\text { massages than they are lacking. }\end{array}$
\end{tabular} &, 669 \\
\hline $\begin{array}{l}\text { No mistakes in their activities } \\
\text { Submission of new products and services }\end{array}$ &, 699 \\
\hline
\end{tabular}

Int.J. Hum. Soc. Dev. Res.

Volume 4, № 1, 2020.41-73 


\begin{tabular}{|l|l|}
\hline Quick results of activities &, 805 \\
\hline The delivery time of products and services is not long &, 652 \\
\hline Product and service ease of production &, 661 \\
\hline Company notification is easy, clear and error free &, 672 \\
\hline Ease of services in other non-business channels &, 700 \\
\hline $\begin{array}{l}\text { Offering new combinations of products and services that better } \\
\text { meet business and service demands }\end{array}$ &, 662 \\
\hline Informing about new campaigns and products &, 670 \\
\hline Making personal campaigns &, 689 \\
\hline $\begin{array}{l}\text { Communication of the operator on special occasions other than the } \\
\text { notification of the product or service (holiday greetings, birthday }\end{array}$ &, 660 \\
\hline Present gifts on special occasions &, 668 \\
\hline Making a lottery campaign &, 654 \\
\hline Care for the appearance of employees &, 670 \\
\hline Employees show sincere efforts to find solutions to problems &, 674 \\
\hline Employees have full knowledge of products and services &, 670 \\
\hline Employees are polite and polite &, 687 \\
\hline Build trust for employees &, 675 \\
\hline
\end{tabular}

When the table is examined 19-20, it is seen that Cronbach Alpha $(\alpha)$ test statistic values of all the scaled items in the questionnaire are among the highly reliable limit values.

Table 21. Frequency Distributions of the Company Average Lifetime Scale

\begin{tabular}{|c|c|c|c|c|c|c|c|c|c|c|c|}
\hline \multirow[t]{2}{*}{$\begin{array}{l}\text { Company } \\
\text { Lifetime }\end{array}$} & \multirow[t]{2}{*}{ Average } & \multicolumn{2}{|c|}{ : } & \multicolumn{2}{|c|}{ 党 } & \multicolumn{2}{|c|}{ 导 } & \multicolumn{2}{|c|}{ 苞 } & \multicolumn{2}{|c|}{$\sum_{i}^{\infty}$} \\
\hline & & $\mathrm{n}$ & $\%$ & $\mathrm{n}$ & $\%$ & $\mathrm{n}$ & $\%$ & $\mathrm{n}$ & $\%$ & 1 & $\%$ \\
\hline
\end{tabular}




\begin{tabular}{|c|c|c|c|c|c|c|c|c|c|c|}
\hline $\begin{array}{l}\text { From time to time rumor } \\
\text { spreads to the business to }\end{array}$ & 23 & 23,0 & 75 & $\mathbf{7 5 , 0}$ & 2 & 2,0 & 0 & 0,0 & 0 & 0,0 \\
\hline $\begin{array}{l}\text { Certain promises (such } \\
\text { as a chef, foreman, etc.) }\end{array}$ & 32 & 32,0 & 68 & 68,0 & 0 & 0,0 & 0 & 0,0 & 0 & 0,0 \\
\hline $\begin{array}{l}\text { Employees who adopt } \\
\text { their own views are } \\
\text { expected to provide a }\end{array}$ & 14 & 14,0 & 86 & 86,0 & 0 & 0,0 & 0 & 0,0 & 0 & 0,0 \\
\hline $\begin{array}{l}\text { It acts politically (as if } \\
\text { showing power), }\end{array}$ & 27 & 27,0 & 73 & $\mathbf{7 3 , 0}$ & 0 & 0,0 & 0 & 0,0 & 0 & 0,0 \\
\hline $\begin{array}{lr}\text { It gives the employees } \\
\text { deliberately different } \\
\text { information }\end{array}$ & 28 & 28,0 & 72 & 72,0 & 0 & 0,0 & 0 & 0,0 & 0 & 0,0 \\
\hline $\begin{array}{l}\text { No mistakes in their } \\
\text { activities }\end{array}$ & 11 & 11,0 & 89 & 89,0 & 0 & 0,0 & 0 & 0,0 & 0 & 0,0 \\
\hline Submission of new & 0 & 0,0 & 100 & 100,0 & 0 & 0,0 & 0 & 0,0 & 0 & 0,0 \\
\hline Quick results of & 55 & 55,0 & 4 & 4,0 & 25 & 25,0 & 16 & 16,0 & 0 & 0,0 \\
\hline $\begin{array}{l}\text { The delivery time of } \\
\text { products and services is }\end{array}$ & 36 & 36,0 & 22 & 22,0 & 42 & 42,0 & 0 & 0,0 & 0 & 0,0 \\
\hline Product and service ease & 43 & 43,0 & 21 & 21,0 & 36 & 36,0 & 0 & 0,0 & 0 & $\overline{0,0}$ \\
\hline $\begin{array}{l}\text { Company notification is } \\
\text { easy, clear and error free }\end{array}$ & 57 & 57,0 & 43 & 43,0 & 0 & 0,0 & 0 & 0,0 & 0 & 0,0 \\
\hline $\begin{array}{l}\text { Ease of services in other } \\
\text { non-business channels }\end{array}$ & 81 & 81,0 & 19 & 19,0 & 0 & 0,0 & 0 & 0,0 & 0 & 0,0 \\
\hline $\begin{array}{l}\text { Offering new } \\
\text { combinations of products } \\
\text { and services that better }\end{array}$ & 29 & 29,0 & 71 & 71,0 & 0 & 0,0 & 0 & 0,0 & 0 & 0,0 \\
\hline $\begin{array}{l}\text { Informing about new } \\
\text { campaigns and products }\end{array}$ & 61 & 61,0 & 39 & 39,0 & 0 & 0,0 & 0 & 0,0 & 0 & 0,0 \\
\hline $\begin{array}{l}\text { Making } \\
\text { camnaigns }\end{array}$ & 6 & 6,0 & 94 & 94,0 & 0 & 0,0 & 0 & 0,0 & 0 & 0,0 \\
\hline $\begin{array}{l}\text { Communication of the } \\
\text { operator on special } \\
\text { occasions other than the } \\
\text { notification of the } \\
\text { nroduct or service }\end{array}$ & 53 & 53,0 & 47 & 47,0 & 0 & 0,0 & 0 & 0,0 & 0 & 0,0 \\
\hline
\end{tabular}

When the table, 21 is examined, It is seen that the company most often participates in the statements on the average life cycle scale survey as "Rarely".

Table 22. Frequency Distributions Related to Company Average Life Span (Continued) 


\begin{tabular}{|c|c|c|c|c|c|c|c|c|c|c|}
\hline \multirow[t]{2}{*}{ Company Average Lifetime } & \multicolumn{2}{|r|}{ ż̀ } & \multicolumn{2}{|r|}{  } & \multicolumn{2}{|c|}{ 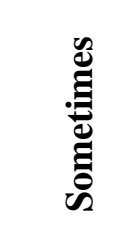 } & \multicolumn{2}{|c|}{$\frac{\vec{\nabla}}{i}$} & \multicolumn{2}{|r|}{$\sum_{i}^{\infty}$} \\
\hline & $\mathrm{n}$ & $\%$ & $\mathrm{n}$ & $\%$ & $\mathrm{n}$ & $\%$ & $\mathrm{n}$ & $\%$ & $\mathrm{n}$ & $\%$ \\
\hline Present gifts on special occasions & 42 & 42,0 & 58 & 58,0 & 0 & 0,0 & 0 & 0,0 & 0 & 0,0 \\
\hline Making a lottery campaign & 26 & 26,0 & 74 & $\mathbf{7 4 , 0}$ & 0 & 0,0 & 0 & 0,0 & 0 & 0,0 \\
\hline $\begin{array}{l}\text { Care for the appearance of } \\
\text { employees }\end{array}$ & 36 & 36,0 & 64 & 64,0 & 0 & 0,0 & 0 & 0,0 & 0 & 0,0 \\
\hline $\begin{array}{l}\text { Employees show sincere efforts } \\
\text { to find solutions to problems }\end{array}$ & 16 & 16,0 & 84 & 84,0 & 0 & 0,0 & 0 & 0,0 & 0 & 0,0 \\
\hline $\begin{array}{l}\text { Employees have full knowledge } \\
\text { of products and services }\end{array}$ & 53 & 53,0 & 47 & 47,0 & 0 & 0,0 & 0 & 0,0 & 0 & 0,0 \\
\hline Employees are polite and polite & 68 & 68,0 & 31 & 31,0 & 1 & 1,0 & 0 & 0,0 & 0 & 0,0 \\
\hline Build trust for employees & 66 & 66,0 & 34 & 34,0 & 0 & 0,0 & 0 & 0,0 & 0 & 0,0 \\
\hline
\end{tabular}

When the table 22 is examined, it is seen that the company most often participates in the statements on the average life cycle scale survey "Rarely".

\section{Company Average Lifetime Scale T-Test and Variance Analysis}

Table 23. Gender and Company Average Lifetime Scale Group Analysis Table Group Analysis

\begin{tabular}{|l|l|l|l|l|l|}
\hline & Gender & N & Mean & $\begin{array}{l}\text { Std. } \\
\text { Deflection }\end{array}$ & Std. Error Mean \\
\hline $\begin{array}{l}\text { Company } \\
\begin{array}{l}\text { Average } \\
\text { Lifetime }\end{array}\end{array}$ & Woman & 56 & 1,6871 &, 19940 &, 02665 \\
\cline { 2 - 6 } & Male & 44 & 1,6858 &, 19932 &, 03005 \\
\hline
\end{tabular}

As can be understood from the above table, the average life expectancy of the company was found to be the average of life expectancy of the firms (56), while the average life expectancy of the male executives $(1,6858)$ of the 44 male managers who contributed to the survey was determined. Women business managers have a higher average perception of the company's average life span.

Table 24. Gender and Company Mean Lifetime Scale Group Analysis Independent Sample T-Test

\section{Independent Sample T-Test}

Levene

Test

Equality of t-test averages 


\begin{tabular}{|c|c|c|c|c|c|c|c|c|c|c|}
\hline & \multirow[t]{2}{*}{$F$} & \multirow[t]{2}{*}{$\begin{array}{l}\text { Sig } \\
\text {. }\end{array}$} & \multirow[t]{2}{*}{$\mathrm{t}$} & \multirow[t]{2}{*}{ Df } & \multirow[t]{2}{*}{$\begin{array}{l}\text { Sig.. } \\
(2- \\
\text { taile } \\
\text { d) }\end{array}$} & \multirow[t]{2}{*}{$\begin{array}{l}\text { Mean } \\
\text { Differe } \\
\text { nce }\end{array}$} & \multirow[t]{2}{*}{$\begin{array}{l}\text { Std. } \\
\text { Error } \\
\text { Differe } \\
\text { nce }\end{array}$} & \multicolumn{2}{|c|}{$\begin{array}{l}95 \% \\
\text { Difference } \\
\text { and } \\
\text { Confidence } \\
\text { Interval }\end{array}$} \\
\hline & & & & & & & & & $\begin{array}{l}\text { Low } \\
\text { er }\end{array}$ & $\begin{array}{l}\text { Uppe } \\
\text { r }\end{array}$ \\
\hline 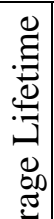 & $\begin{array}{l}\text { Assumi } \\
\text { ng } \\
\text { equal } \\
\text { varianc } \\
\text { e }\end{array}$ & $\begin{array}{l}, 0 \\
21\end{array}$ & $\begin{array}{l}, 8 \\
89\end{array}$ & $\begin{array}{l}, 0 \\
34\end{array}$ & 99 & $\begin{array}{l}, 97 \\
4\end{array}$ & ,00135 &, 04015 & $\begin{array}{l}- \\
, 078 \\
36\end{array}$ & $\begin{array}{l}, 081 \\
04\end{array}$ \\
\hline  & $\begin{array}{l}\text { Do not } \\
\text { assume } \\
\text { equal } \\
\text { varianc } \\
\text { e }\end{array}$ & & & $\begin{array}{l}, 0 \\
34\end{array}$ & $\begin{array}{l}92,4 \\
99\end{array}$ & $\begin{array}{l}, 97 \\
4\end{array}$ & ,00135 & ,04017 & $\begin{array}{l}- \\
, 078 \\
42\end{array}$ & $\begin{array}{l}, 081 \\
10\end{array}$ \\
\hline
\end{tabular}

Whether there is a meaningful difference between the opinions of male and female business managers about the average life expectancy of the company is shown in the table above. (2-tailed) we can understand the value of. When the value of significance is less than 0.05 , there is a meaningful difference between the two groups. Since the significance value of the analysis we conducted is $0,974>0,05$, it is understood that the average life expectancy of the company is not significantly different between male and female business managers. $\mathrm{H}_{9}$ hypothesis is rejected because male and female business managers' mean life span opinions of the company are significant value $p>0.05$. In other words, the average life expectancy of male and female business managers does not differ significantly in terms of gender segmentation.

Table 25. Age and Company Average Lifetime Descriptive Statistics Table

\begin{tabular}{|c|c|c|c|c|c|c|c|c|}
\hline \multicolumn{9}{|c|}{ Descriptive Statistics } \\
\hline \multicolumn{9}{|c|}{ Company Average Lifetime } \\
\hline \multirow[t]{3}{*}{ Age } & \multirow[t]{3}{*}{$\mathrm{N}$} & \multirow[t]{3}{*}{ Mean } & \multirow{3}{*}{$\begin{array}{l}\text { Std. } \\
\text { Deflectio } \\
n\end{array}$} & \multirow{3}{*}{$\begin{array}{l}\text { Std. } \\
\text { Error }\end{array}$} & \multirow{2}{*}{\multicolumn{2}{|c|}{$\begin{array}{ll}\text { Mean } & 95 \% \\
\text { confidence } & \\
\text { interval } & \\
\end{array}$}} & \multirow{3}{*}{ Min } & \multirow{3}{*}{$\begin{array}{l}\text { Max } \\
\text {. }\end{array}$} \\
\hline & & & & & & & & \\
\hline & & & & & $\begin{array}{l}\text { Lower } \\
\text { Limit }\end{array}$ & $\begin{array}{l}\text { Upper } \\
\text { Limit }\end{array}$ & & \\
\hline $\begin{array}{l}\text { betwee } \\
\text { n 21-30 } \\
\text { years }\end{array}$ & 12 & $\begin{array}{l}1,804 \\
4\end{array}$ & ,11944 & $\begin{array}{l}, 0344 \\
9\end{array}$ & $\begin{array}{l}1,728 \\
6\end{array}$ & $\begin{array}{l}1,880 \\
3\end{array}$ & $\begin{array}{l}1,6 \\
2\end{array}$ & $\begin{array}{l}1,9 \\
6\end{array}$ \\
\hline $\begin{array}{l}\text { betwee } \\
\text { n } 31-40 \\
\text { years }\end{array}$ & 10 & $\begin{array}{l}1,674 \\
0\end{array}$ & , 16683 & $\begin{array}{l}, 0527 \\
6\end{array}$ & $\begin{array}{l}1,554 \\
7\end{array}$ & $\begin{array}{l}1,793 \\
4\end{array}$ & $\begin{array}{l}1,4 \\
4\end{array}$ & $\begin{array}{l}1,8 \\
7\end{array}$ \\
\hline $\begin{array}{l}\text { betwee } \\
\text { n } 41-50 \\
\text { years }\end{array}$ & 18 & $\begin{array}{l}1,727 \\
2\end{array}$ &, 16527 & $\begin{array}{l}, 0389 \\
1\end{array}$ & $\begin{array}{l}1,645 \\
0\end{array}$ & $\begin{array}{l}1,809 \\
3\end{array}$ & $\begin{array}{l}1,4 \\
0\end{array}$ & $\begin{array}{l}1,9 \\
6\end{array}$ \\
\hline
\end{tabular}




\begin{tabular}{|l|l|l|l|l|l|l|l|l|}
\hline $\begin{array}{l}\text { betwee } \\
\text { n 51-60 } \\
\text { years }\end{array}$ & 31 & $\begin{array}{l}1,542 \\
9\end{array}$ &, 21996 & $\begin{array}{l}, 0395 \\
1\end{array}$ & $\begin{array}{l}1,462 \\
2\end{array}$ & $\begin{array}{l}1,623 \\
6\end{array}$ & $\begin{array}{l}1,2 \\
7\end{array}$ & $\begin{array}{l}1,9 \\
1\end{array}$ \\
\hline $\begin{array}{l}60 \\
\text { years } \\
\text { and } \\
\text { over }\end{array}$ & 29 & $\begin{array}{l}1,770 \\
7\end{array}$ &, 13745 & $\begin{array}{l}0255 \\
3\end{array}$ & $\begin{array}{l}1,718 \\
4\end{array}$ & $\begin{array}{l}1,823 \\
0\end{array}$ & $\begin{array}{l}1,4 \\
0\end{array}$ & $\begin{array}{l}1,9 \\
6\end{array}$ \\
\hline Toplam & $\begin{array}{l}10 \\
0\end{array}$ & $\begin{array}{l}1,686 \\
6\end{array}$ &, 19837 & $\begin{array}{l}0198 \\
5\end{array}$ & $\begin{array}{l}1,647 \\
3\end{array}$ & $\begin{array}{l}1,726 \\
0\end{array}$ & $\begin{array}{l}1,2 \\
7\end{array}$ & $\begin{array}{l}1,9 \\
6\end{array}$ \\
\hline
\end{tabular}

Business managers with ages 21-30 have the highest average corporate life expectancy $(1,8044)$, while managers with age groups 51-60 have the lowest average $(1,5428)$ of the company's average life expectancy.

Table 26. Age and Company Average Life Span Anova Table

\begin{tabular}{|c|c|c|c|c|c|c|}
\hline \multicolumn{7}{|l|}{ ANOVA } \\
\hline \multicolumn{7}{|c|}{ Company Average Lifetime } \\
\hline & $\begin{array}{l}\text { Sum of } \\
\text { squares }\end{array}$ & df & $\begin{array}{l}\text { Squares } \\
\text { average }\end{array}$ & $\mathrm{F}$ & Sig. & Post-Hoc ve Scheffe \\
\hline $\begin{array}{l}\text { Between } \\
\text { groups }\end{array}$ & 1,044 & 4 & ,263 & 8,691 & ,002 & $\begin{array}{lll}\text { between } & 21-30 & \text { years, } \\
\text { between } & 41-50 & \text { years, }\end{array}$ \\
\hline $\begin{array}{l}\text { Within } \\
\text { groups }\end{array}$ & 2,853 & 95 & ,032 & & & $\begin{array}{l}\text { between } 51-60 \text { years, } 60 \\
\text { years and over> between } 31-\end{array}$ \\
\hline Total & 3,896 & 99 & & & & 40 years \\
\hline
\end{tabular}

According to $\mathrm{F}$ test result at $95 \%$ confidence level; The mean life span of company managers' significance level was found as $\mathrm{p}=0.002<0.05$. The $\mathrm{H}_{10}$ hypothesis was accepted when the significance level of the opinion of the business managers about the life expectancy of the company was $\mathrm{p}<0.05$. As a result of the post-hoc and scheffe analysis on which group or groups the significance arises from; The age group of 21-30 years, 41-50 years, 51-60 years, 60 years and over participates higher than other groups (31-40 years).

Table 27. Marital Status and Company Average Lifetime Descriptive Statistics

Table

Tanımlayıcı İstatistik

Company Average Lifetime

\begin{tabular}{|c|c|c|c|c|c|c|c|c|}
\hline \multirow[t]{2}{*}{$\begin{array}{l}\text { Marital } \\
\text { Status }\end{array}$} & \multirow[t]{2}{*}{$\mathrm{N}$} & \multirow[t]{2}{*}{ Mean } & \multirow[t]{2}{*}{$\begin{array}{l}\text { Std. } \\
\text { Deflectio } \\
n\end{array}$} & \multirow[t]{2}{*}{$\begin{array}{l}\text { Std. } \\
\text { Error }\end{array}$} & \multicolumn{2}{|c|}{$\begin{array}{ll}\text { Mean } & 95 \% \\
\text { confidence } & \\
\text { interval } & \end{array}$} & \multirow[t]{2}{*}{ Min. } & \multirow[t]{2}{*}{ Max } \\
\hline & & & & & $\begin{array}{l}\text { Lower } \\
\text { Limit }\end{array}$ & $\begin{array}{l}\text { Upper } \\
\text { Limit }\end{array}$ & & \\
\hline $\begin{array}{l}\text { The } \\
\text { married }\end{array}$ & 18 & $\begin{array}{l}1,876 \\
9\end{array}$ & ,08066 & $\begin{array}{l}, 0190 \\
2\end{array}$ & $\begin{array}{l}1,836 \\
8\end{array}$ & $\begin{array}{l}1,917 \\
0\end{array}$ & $\begin{array}{l}1,7 \\
1\end{array}$ & 1,97 \\
\hline Single & 47 & $\begin{array}{l}1,649 \\
5\end{array}$ & , 18656 & $\begin{array}{l}, 0272 \\
2\end{array}$ & $\begin{array}{l}1,594 \\
7\end{array}$ & $\begin{array}{l}1,704 \\
3\end{array}$ & $\begin{array}{l}1,3 \\
1\end{array}$ & 1,92 \\
\hline Divorce & 11 & 1,723 & ,10699 & ,0322 & 1,651 & 1,795 & 1,6 & 1,92 \\
\hline
\end{tabular}




\begin{tabular}{|l|l|l|l|l|l|l|l|l|}
\hline $\mathrm{d}$ & & 4 & & 7 & 5 & 3 & 2 & \\
\hline $\begin{array}{l}\text { His wife } \\
\text { passed } \\
\text { away }\end{array}$ & 24 & $\begin{array}{l}1,599 \\
7\end{array}$ &, 22428 & $\begin{array}{l}, 0457 \\
9\end{array}$ & $\begin{array}{l}1,505 \\
0\end{array}$ & $\begin{array}{l}1,694 \\
4\end{array}$ & $\begin{array}{l}1,2 \\
7\end{array}$ & 1,97 \\
\hline Total & $\begin{array}{l}10 \\
0\end{array}$ & $\begin{array}{l}1,686 \\
6\end{array}$ &, 19837 & $\begin{array}{l}, 0198 \\
5\end{array}$ & $\begin{array}{l}1,647 \\
3\end{array}$ & $\begin{array}{l}1,726 \\
0\end{array}$ & $\begin{array}{l}1,2 \\
7\end{array}$ & 1,97 \\
\hline
\end{tabular}

Marital status groups have the highest average level of married enterprise managers 'average life expectancy $(1,8769)$, while marital status groups have the lowest average $(1,5997)$ of married executive managers' views on company life expectancy.

Table 28. Marital Status and Company Average Life Span Anova Table

\begin{tabular}{|c|c|c|c|c|c|c|}
\hline \multicolumn{7}{|l|}{ ANOVA } \\
\hline \multicolumn{7}{|c|}{ Company Average Lifetime } \\
\hline & $\begin{array}{l}\text { Sum of } \\
\text { squares }\end{array}$ & $\mathrm{df}$ & $\begin{array}{l}\text { Squares } \\
\text { average }\end{array}$ & $\mathrm{F}$ & Sig. & $\begin{array}{ll}\text { Post-Hoc } & \text { ve } \\
\text { Scheffe } & \end{array}$ \\
\hline $\begin{array}{l}\text { Between } \\
\text { groups }\end{array}$ & ,914 & 3 & ,305 & 9,792 & ,001 & \multirow{3}{*}{$\begin{array}{l}\text { The married, } \\
\text { Single, His wife } \\
\text { passed away > } \\
\text { Divorced }\end{array}$} \\
\hline $\begin{array}{l}\text { Within } \\
\text { groups }\end{array}$ & 2,984 & 96 &, 032 & & & \\
\hline Total & 3,896 & 99 & & & & \\
\hline
\end{tabular}

According to $\mathrm{F}$ test result at $95 \%$ confidence level; The opinions of business managers on the average life span of the company were determined as significance value $\mathrm{p}=$ $0.001<0.05$. The $\mathrm{H}_{11}$ hypothesis was accepted because of the significance value of the opinion of business managers regarding the average life span of the company is $p<0.05$. As a result of the post-hoc and scheffe analysis on which group or groups the significance arises from; Married, single, married status group participates higher than other groups (Divorced).

Table 29. Educational Status and Company Average Lifetime Descriptive Statistics

Table

\begin{tabular}{|c|c|c|c|c|c|c|c|c|}
\hline \multicolumn{9}{|c|}{ Tanımlayıc İstatistik } \\
\hline \multicolumn{9}{|c|}{ Company Average Lifetime } \\
\hline \multirow[t]{2}{*}{$\begin{array}{l}\text { Education } \\
\text { al Status }\end{array}$} & \multirow[t]{2}{*}{$\mathrm{N}$} & \multirow[t]{2}{*}{ Mean } & \multirow[t]{2}{*}{$\begin{array}{l}\text { Std. } \\
\text { Deflectio } \\
n\end{array}$} & \multirow[t]{2}{*}{$\begin{array}{l}\text { Std. } \\
\text { Error }\end{array}$} & \multicolumn{2}{|c|}{$\begin{array}{ll}\text { Mean } & 95 \% \\
\text { confidence } & \\
\text { interval } & \end{array}$} & \multirow[t]{2}{*}{ Min } & \multirow[t]{2}{*}{ Max } \\
\hline & & & & & $\begin{array}{l}\text { Lower } \\
\text { Limit }\end{array}$ & $\begin{array}{l}\text { Upper } \\
\text { Limit }\end{array}$ & & \\
\hline $\begin{array}{l}\text { Primary } \\
\text { school }\end{array}$ & 41 & $\begin{array}{l}1,619 \\
4\end{array}$ & , 18235 & $\begin{array}{l}, 0284 \\
9\end{array}$ & $\begin{array}{l}1,561 \\
8\end{array}$ & $\begin{array}{l}1,677 \\
0\end{array}$ & $\begin{array}{l}1,2 \\
7\end{array}$ & $\begin{array}{l}1,9 \\
2\end{array}$ \\
\hline $\begin{array}{l}\text { Middle } \\
\text { School }\end{array}$ & 16 & $\begin{array}{l}1,728 \\
4\end{array}$ & , 19477 & $\begin{array}{l}, 0487 \\
0\end{array}$ & $\begin{array}{l}1,624 \\
6\end{array}$ & $\begin{array}{l}1,832 \\
1\end{array}$ & $\begin{array}{l}1,4 \\
0\end{array}$ & $\begin{array}{l}1,9 \\
7\end{array}$ \\
\hline $\begin{array}{l}\text { High } \\
\text { school }\end{array}$ & 19 & $\begin{array}{l}1,794 \\
2\end{array}$ & , 15464 & $\begin{array}{l}, 0354 \\
9\end{array}$ & $\begin{array}{l}1,719 \\
6\end{array}$ & $\begin{array}{l}1,868 \\
7 \\
\end{array}$ & $\begin{array}{l}1,4 \\
0 \\
\end{array}$ & $\begin{array}{l}1,9 \\
7 \\
\end{array}$ \\
\hline Associate & 7 & $\begin{array}{l}1,801 \\
3\end{array}$ & , 14390 & $\begin{array}{l}, 0543 \\
9\end{array}$ & $\begin{array}{l}1,668 \\
3\end{array}$ & $\begin{array}{l}1,934 \\
4\end{array}$ & $\begin{array}{l}1,6 \\
2\end{array}$ & $\begin{array}{l}1,9 \\
7\end{array}$ \\
\hline
\end{tabular}




\begin{tabular}{|l|l|l|l|l|l|l|l|l|}
\hline $\begin{array}{l}\text { Bachelor / } \\
\text { University }\end{array}$ & 8 & $\begin{array}{l}1,440 \\
3\end{array}$ &, 16008 & $\begin{array}{l}, 0566 \\
0\end{array}$ & $\begin{array}{l}1,306 \\
5\end{array}$ & $\begin{array}{l}1,574 \\
1\end{array}$ & $\begin{array}{l}1,3 \\
1\end{array}$ & $\begin{array}{l}1,7 \\
9\end{array}$ \\
\hline Master & 9 & $\begin{array}{l}1,821 \\
4\end{array}$ &, 10527 & $\begin{array}{l}, 0351 \\
0\end{array}$ & $\begin{array}{l}1,740 \\
4\end{array}$ & $\begin{array}{l}1,902 \\
3\end{array}$ & $\begin{array}{l}1,7 \\
1\end{array}$ & $\begin{array}{l}1,9 \\
7\end{array}$ \\
\hline Total & $\begin{array}{l}10 \\
0\end{array}$ & $\begin{array}{l}1,686 \\
6\end{array}$ &, 19837 & $\begin{array}{l}, 0198 \\
5\end{array}$ & $\begin{array}{l}1,647 \\
3\end{array}$ & $\begin{array}{l}1,726 \\
0\end{array}$ & $\begin{array}{l}1,2 \\
7\end{array}$ & $\begin{array}{l}1,9 \\
7\end{array}$ \\
\hline
\end{tabular}

While the average of the managers of graduate business managers in the education groups is the highest level of the average life expectancy of the company $(1,8214)$, the average level of the average life expectancy of the managers of the education groups is bachelor / university $(1,4403)$.

Table 30. Education and Company Average Life Span Anova Table

\begin{tabular}{|c|c|c|c|c|c|c|}
\hline \multicolumn{7}{|l|}{ ANOVA } \\
\hline \multicolumn{7}{|c|}{ Company Average Lifetime } \\
\hline & $\begin{array}{l}\text { Sum of } \\
\text { squares }\end{array}$ & df & $\begin{array}{l}\text { Squares } \\
\text { average }\end{array}$ & $\mathrm{F}$ & Sig. & $\begin{array}{l}\text { Post-Hoc ve } \\
\text { Scheffe }\end{array}$ \\
\hline $\begin{array}{l}\text { Between } \\
\text { groups }\end{array}$ & 1,175 & 5 & ,236 & 8,108 & ,001 & \multirow{3}{*}{$\begin{array}{l}\text { Bachelor } \\
\text { University, } \\
\text { Master>Primary } \\
\text { school } \\
\text { Middle School, } \\
\text { High school, } \\
\text { Associate }\end{array}$} \\
\hline $\begin{array}{l}\text { Within } \\
\text { groups }\end{array}$ & 2,723 & 94 &, 030 & & & \\
\hline Total & 3,896 & 99 & & & & \\
\hline
\end{tabular}

According to $\mathrm{F}$ test result at $95 \%$ confidence level; meaningful value of business managers' opinions about company life expectancy was found as $p=0.001<0.05$. The $\mathrm{H}_{12}$ hypothesis was accepted because the significance level of the opinion of the business managers about the life expectancy of the company is $\mathrm{p}<0.05$.

Table 31. Working Time at Company and Company Average Lifetime Descriptive Statistics Table

\begin{tabular}{|c|c|c|c|c|c|c|c|c|}
\hline \multicolumn{9}{|c|}{ Descriptive Statistics } \\
\hline \multicolumn{9}{|c|}{ Company Average Lifetime } \\
\hline \multirow{3}{*}{$\begin{array}{l}\text { Working } \\
\text { Time in } \\
\text { Compan } \\
\text { y }\end{array}$} & \multirow[t]{3}{*}{$\mathrm{N}$} & \multirow[t]{3}{*}{ Mean } & \multirow{3}{*}{$\begin{array}{l}\text { Std. } \\
\text { Deflectio } \\
\mathrm{n}\end{array}$} & \multirow{3}{*}{$\begin{array}{l}\text { Std. } \\
\text { Error }\end{array}$} & \multirow{2}{*}{\multicolumn{2}{|c|}{$\begin{array}{ll}\text { Mean } & 95 \% \\
\text { confidence } & \\
\text { interval } & \\
\end{array}$}} & \multirow{3}{*}{ Min } & \multirow{3}{*}{ Max } \\
\hline & & & & & & & & \\
\hline & & & & & $\begin{array}{l}\text { Lower } \\
\text { Limit }\end{array}$ & $\begin{array}{l}\text { Upper } \\
\text { Limit }\end{array}$ & & \\
\hline $1-5$ & 60 & 1,763 & ,14263 & 0184 & 1,726 & 1,710 & 1,4 & 1,9 \\
\hline year & & $\frac{1}{15(0)}$ & & 2 & 3 & 0 & 0 & 7 \\
\hline 6-10 & 38 & 1,569 & 22143 & ,0359 & 1,497 & 1,642 & 1,2 & 1,9 \\
\hline
\end{tabular}




\begin{tabular}{|l|l|l|l|l|l|l|l|l|}
\hline year & & 9 & & 3 & 1 & 7 & 7 & 2 \\
\hline $\begin{array}{l}\text { 21 year } \\
\text { and } \\
\text { over }\end{array}$ & 2 & $\begin{array}{l}1,608 \\
8\end{array}$ &, 00000 & $\begin{array}{l}, 0000 \\
0\end{array}$ & $\begin{array}{l}1,608 \\
8\end{array}$ & $\begin{array}{l}1,608 \\
8\end{array}$ & $\begin{array}{l}1,6 \\
2\end{array}$ & $\begin{array}{l}1,6 \\
2\end{array}$ \\
\hline Total & $\begin{array}{l}10 \\
0\end{array}$ & $\begin{array}{l}1,686 \\
6\end{array}$ &, 19837 & $\begin{array}{l}, 0198 \\
5\end{array}$ & $\begin{array}{l}1,647 \\
3\end{array}$ & $\begin{array}{l}1,726 \\
0\end{array}$ & $\begin{array}{l}1,2 \\
7\end{array}$ & $\begin{array}{l}1,9 \\
7\end{array}$ \\
\hline
\end{tabular}

In the company, the average life expectancy of company managers (1-5631) is the highest level among the business managers of the study period groups, while the average life expectancy of the company managers is 6-10 years in the company $(1,5699)$ lowest level.

Table 32. Working Time at Company and Company Average Life Time Anova Table

\begin{tabular}{|c|c|c|c|c|c|c|}
\hline \multicolumn{7}{|l|}{ ANOVA } \\
\hline \multicolumn{7}{|c|}{ Company Average Lifetime } \\
\hline & $\begin{array}{l}\text { Sum of } \\
\text { squares }\end{array}$ & df & $\begin{array}{l}\text { Squares } \\
\text { average }\end{array}$ & $\mathrm{F}$ & Sig. & $\begin{array}{ll}\text { Post-Hoc } & \text { ve } \\
\text { Scheffe } & \end{array}$ \\
\hline $\begin{array}{l}\text { Between } \\
\text { groups }\end{array}$ & ,882 & 2 & ,442 & 14,181 & 001 & \multirow{3}{*}{$\begin{array}{l}1-5 \text { year, 6-10 } \\
\text { year>21 year and } \\
\text { over }\end{array}$} \\
\hline $\begin{array}{l}\text { Within } \\
\text { groups }\end{array}$ & 3,015 & 97 &, 032 & & & \\
\hline Total & 3,896 & 99 & & & & \\
\hline
\end{tabular}

According to $\mathrm{F}$ test result at $95 \%$ confidence level; The significance level of the opinion of the business managers regarding the average life span of the company was found to be $\mathrm{p}=0.001<0.05$. The hypothesis $\mathrm{H}_{13}$ is accepted if the opinion of the business managers about the life expectancy of the company is $\mathrm{p}<0.05$.

Table 33. Company Type and Company Average Lifetime Descriptive Statistics

Table

\begin{tabular}{|c|c|c|c|c|c|c|c|c|}
\hline \multicolumn{9}{|c|}{ Descriptive Statistical } \\
\hline \multicolumn{9}{|c|}{ Company Average Lifetime } \\
\hline \multirow{3}{*}{$\begin{array}{l}\text { Company } \\
\text { Type }\end{array}$} & \multirow[t]{3}{*}{$\mathrm{N}$} & \multirow[t]{3}{*}{ Mean } & \multirow{3}{*}{$\begin{array}{l}\text { Std. } \\
\text { Deflectio } \\
\mathrm{n}\end{array}$} & \multirow{3}{*}{$\begin{array}{l}\text { Std. } \\
\text { Error }\end{array}$} & \multirow{2}{*}{\multicolumn{2}{|c|}{$\begin{array}{l}\text { Mean } 95 \% \\
\text { confidence } \\
\text { interval }\end{array}$}} & \multirow{3}{*}{ Min } & \multirow{3}{*}{ Max } \\
\hline & & & & & & & & \\
\hline & & & & & $\begin{array}{l}\text { Lower } \\
\text { Limit }\end{array}$ & $\begin{array}{l}\text { Upper } \\
\text { Limit }\end{array}$ & & \\
\hline $\begin{array}{l}\text { Joint Stock } \\
\text { Company } \\
\text { (Inc) }\end{array}$ & 23 & $\begin{array}{l}1,606 \\
9\end{array}$ & ,23579 & $\begin{array}{l}, 0491 \\
7\end{array}$ & $\begin{array}{l}1,504 \\
9\end{array}$ & $\begin{array}{l}1,708 \\
9\end{array}$ & $\begin{array}{l}1,2 \\
7\end{array}$ & $\begin{array}{l}1,9 \\
7\end{array}$ \\
\hline $\begin{array}{l}\text { Limited } \\
\text { company } \\
\text { (Co. Ltd.) } \\
\end{array}$ & 75 & $\begin{array}{l}1,703 \\
9\end{array}$ &, 17852 & $\begin{array}{l}, 0206 \\
2\end{array}$ & $\begin{array}{l}1,662 \\
8\end{array}$ & $\begin{array}{l}1,744 \\
9\end{array}$ & $\begin{array}{l}1,3 \\
1\end{array}$ & $\begin{array}{l}1,9 \\
7\end{array}$ \\
\hline
\end{tabular}




\begin{tabular}{|l|l|l|l|l|l|l|l|l|}
\hline $\begin{array}{l}\text { Cooperativ } \\
\text { e company }\end{array}$ & 2 & $\begin{array}{l}1,956 \\
6\end{array}$ &, 00000 & $\begin{array}{l}, 0000 \\
0\end{array}$ & $\begin{array}{l}1,956 \\
6\end{array}$ & $\begin{array}{l}1,956 \\
6\end{array}$ & $\begin{array}{l}1,9 \\
7\end{array}$ & $\begin{array}{l}1,9 \\
7\end{array}$ \\
\hline Total & 10 & $\begin{array}{l}1,686 \\
6\end{array}$ &, 19837 & $\begin{array}{l}, 0198 \\
5\end{array}$ & $\begin{array}{l}1,647 \\
3\end{array}$ & $\begin{array}{l}1,726 \\
0\end{array}$ & $\begin{array}{l}1,2 \\
7\end{array}$ & $\begin{array}{l}1,9 \\
7\end{array}$ \\
\hline
\end{tabular}

While the average life expectancy of company managers is the highest level (1.9566), the average of life expectancy of company managers is (1.6069), while that of company type group cooperatives is the highest low level.

Table 34. Company Type and Company Average Life Span Anova Table

\begin{tabular}{|c|c|c|c|c|c|c|}
\hline \multicolumn{7}{|c|}{ ANOVA } \\
\hline \multicolumn{7}{|c|}{ Şirket Mean Yaşam Süresi } \\
\hline  & $\begin{array}{l}\text { Sum of } \\
\text { squares }\end{array}$ & df & $\begin{array}{l}\text { Squares } \\
\text { average }\end{array}$ & $\mathrm{F}$ & Sig. & $\begin{array}{l}\text { Post-Hoc ve } \\
\text { Scheffe }\end{array}$ \\
\hline $\begin{array}{l}\text { Between } \\
\text { groups }\end{array}$ & ,315 & 2 &, 158 & 4,257 & ,018 & \multirow{3}{*}{  } \\
\hline $\begin{array}{l}\text { Within } \\
\text { groups }\end{array}$ & 3,582 & 97 &, 038 & & & \\
\hline Total & 3,896 & 99 & & & & \\
\hline
\end{tabular}

According to $\mathrm{F}$ test result at $95 \%$ confidence level; The significance value of the opinion of the business managers about the average life span of the company was found as $\mathrm{p}=0,018<0,05 . \mathrm{H}_{14}$ hypothesis was accepted because the opinion of business managers about the average life span of the company is $\mathrm{p}<0.05$.

Table 35. Number of Employees in the Company and Company Average Lifetime Scale Group Analysis Table

\begin{tabular}{|c|c|c|c|c|c|}
\hline \multicolumn{6}{|c|}{ Group Analysis } \\
\hline \multirow{3}{*}{$\begin{array}{l}\text { Company } \\
\text { Average } \\
\text { Lifetime }\end{array}$} & $\begin{array}{l}\text { Number of } \\
\text { Employees } \\
\text { in the } \\
\text { Company }\end{array}$ & $\mathrm{N}$ & Mean & $\begin{array}{l}\text { Std. } \\
\text { Deflection }\end{array}$ & Std. Error Mean \\
\hline & $\begin{array}{l}0-9 \\
\text { between }\end{array}$ & 32 & 1,7773 & ,12677 &, 02242 \\
\hline & $\begin{array}{l}10-49 \\
\text { between }\end{array}$ & 68 & 1,6440 & ,21198 &, 02572 \\
\hline
\end{tabular}

As can be understood from the above table, while the average number of managers in the 32 companies participating in the survey is between 0 and $9(1,7773)$, the number of the managers in the 68 companies contributing to the study is between 10 and 49, and the average of their scores $(1,6440)$. Business managers whose number of employees is between 0 and 9 in the company have higher levels of company life expectancy. 
Table 36. Number of Employees in the Company and Company Average Lifetime Scale Group Analysis Independent Sample T-Test

\begin{tabular}{|c|c|c|c|c|c|c|c|c|c|c|}
\hline \multicolumn{11}{|c|}{ Independent Sample T-Test } \\
\hline & & \multicolumn{2}{|c|}{$\begin{array}{l}\text { Levene } \\
\text { Test }\end{array}$} & \multicolumn{7}{|c|}{ Equality of t-test averages } \\
\hline & & \multirow[t]{2}{*}{$\mathrm{F}$} & \multirow[t]{2}{*}{ Sig } & \multirow[t]{2}{*}{$\mathrm{t}$} & \multirow[t]{2}{*}{ Df } & \multirow[t]{2}{*}{$\begin{array}{l}\text { Sig. } \\
.(2- \\
\text { tail } \\
\text { ed })\end{array}$} & \multirow[t]{2}{*}{$\begin{array}{l}\text { Mean } \\
\text { Differe } \\
\text { nce }\end{array}$} & \multirow[t]{2}{*}{$\begin{array}{l}\text { Std. } \\
\text { Error } \\
\text { Differe } \\
\text { nce }\end{array}$} & \multicolumn{2}{|c|}{$\begin{array}{l}95 \% \\
\text { Difference } \\
\text { and } \\
\text { Confidence } \\
\text { Interval }\end{array}$} \\
\hline & & & & & & & & & $\begin{array}{l}\text { Low } \\
\text { er }\end{array}$ & $\begin{array}{l}\text { Upp } \\
\text { er }\end{array}$ \\
\hline 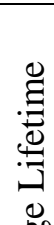 & $\begin{array}{l}\text { Assum } \\
\text { ing } \\
\text { equal } \\
\text { varian } \\
\text { ce }\end{array}$ & $\begin{array}{l}18,9 \\
24\end{array}$ & $\begin{array}{l}, 0 \\
01\end{array}$ & $\begin{array}{l}3,2 \\
88\end{array}$ & 99 & $\begin{array}{l}, 00 \\
2\end{array}$ & $\begin{array}{l}, 1333 \\
2\end{array}$ & $\begin{array}{l}, 0405 \\
7\end{array}$ & $\begin{array}{l}, 052 \\
83\end{array}$ & $\begin{array}{l}, 213 \\
82\end{array}$ \\
\hline 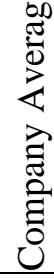 & $\begin{array}{l}\text { Do not } \\
\text { assum } \\
\mathrm{e} \\
\text { equal } \\
\text { varian } \\
\text { ce }\end{array}$ & & & $\begin{array}{l}3,9 \\
10\end{array}$ & $\begin{array}{l}92,3 \\
10\end{array}$ & $\begin{array}{l}, 00 \\
1\end{array}$ & $\begin{array}{l}, 1333 \\
2\end{array}$ & $\begin{array}{l}, 0341 \\
1\end{array}$ & $\begin{array}{l}, 065 \\
60\end{array}$ & $\begin{array}{l}, 201 \\
05\end{array}$ \\
\hline
\end{tabular}

According to the number of employees in the company, whether there is a meaningful difference between the opinions of the business managers on the average life expectancy of the company is shown in the table above. (2-tailed) we can understand the value of. When the value of significance is less than 0.05 , there is a meaningful difference between the two groups. The significance value of our analysis is 0.002 $<0.05$ and it is understood that the average life expectancy of company managers differ significantly according to the number of employees in the company. According to the number of employees in the company, the H15 hypothesis is accepted because the meaning of the company average life cycle views is $p<0,05$. In other words, in terms of number of employees in the company, the average life span of company managers differ significantly.

Table 37. Community Survey of Competitiveness and Company Average Lifetime Scale Group Analysis Table

\begin{tabular}{|l|l|l|l|l|l|}
\hline Group Analysis \\
\hline \multirow{3}{*}{$\begin{array}{l}\text { Company } \\
\text { Average } \\
\text { Lifetime }\end{array}$} & $\begin{array}{l}\text { The Competitive Situation of } \\
\text { the Region in which the } \\
\text { Operator }\end{array}$ & N & Mean & $\begin{array}{l}\text { Std. } \\
\text { Deflection }\end{array}$ & $\begin{array}{l}\text { Std. } \\
\text { Error } \\
\text { Mean }\end{array}$ \\
\cline { 2 - 7 } & There is no competition & 14 & 1,6554 &, 13371 &, 03574 \\
\cline { 2 - 7 } & There is some competition & 86 & 1,6917 &, 20715 &, 02235 \\
\hline
\end{tabular}

As the table above indicates, there are 14 companies participating in the survey. There is no competition in the environment. The average life expectancy of the company is $(1,6554)$ while the average of the 86 managers who have contributed to the study is 
somewhat competitive. and the average of the opinion (1.6917). Business managers who say that there is some competition in the environment where the business is located have a higher average level of corporate life span.

Table 38. Civilian Competition Status and Company Average Lifetime Scale for Operational Analysis Group Analysis Independent Sample T-Test

\begin{tabular}{|c|c|c|c|c|c|c|c|c|c|c|}
\hline \multicolumn{11}{|c|}{ Independent Sample T-Test } \\
\hline & & \multicolumn{2}{|c|}{$\begin{array}{l}\text { Levene } \\
\text { Test }\end{array}$} & \multicolumn{7}{|c|}{ Equality of t-test averages } \\
\hline & & \multirow[t]{2}{*}{$\mathrm{F}$} & \multirow[t]{2}{*}{$\begin{array}{l}\text { Sig } \\
\text {. }\end{array}$} & \multirow[t]{2}{*}{$\mathrm{t}$} & \multirow[t]{2}{*}{$\overline{\mathrm{Df}}$} & \multirow[t]{2}{*}{$\begin{array}{l}\text { Sig. } \\
(2- \\
\text { taile } \\
\text { d) }\end{array}$} & \multirow[t]{2}{*}{$\begin{array}{l}\text { Mean } \\
\text { Differe } \\
\text { nce }\end{array}$} & \multirow[t]{2}{*}{$\begin{array}{l}\text { Std. } \\
\text { Error } \\
\text { Differe } \\
\text { nce }\end{array}$} & \multicolumn{2}{|c|}{$\begin{array}{l}95 \% \\
\text { Difference } \\
\text { and } \\
\text { Confidence } \\
\text { Interval }\end{array}$} \\
\hline & & & & & & & & & $\begin{array}{l}\text { Low } \\
\text { er }\end{array}$ & $\begin{array}{l}\text { Uppe } \\
\text { r }\end{array}$ \\
\hline 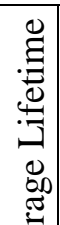 & $\begin{array}{l}\text { Assum } \\
\text { ing } \\
\text { equal } \\
\text { varianc } \\
\text { e }\end{array}$ & $\begin{array}{l}4,3 \\
09\end{array}$ & $\begin{array}{l}, 0 \\
42\end{array}$ & $\begin{array}{l}- \\
, 6 \\
35\end{array}$ & 99 & $\begin{array}{l}, 52 \\
9\end{array}$ & $\begin{array}{l}- \\
, 03634\end{array}$ & 05735 & $\begin{array}{l}- \\
, 150 \\
13\end{array}$ & $\begin{array}{l}, 077 \\
47\end{array}$ \\
\hline 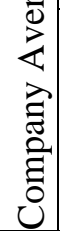 & $\begin{array}{l}\text { Do not } \\
\text { assume } \\
\text { equal } \\
\text { varianc } \\
\text { e }\end{array}$ & & & $\begin{array}{l}- \\
, 8 \\
63\end{array}$ & $\begin{array}{l}24,5 \\
71\end{array}$ & $\begin{array}{l}, 39 \\
8\end{array}$ & $\begin{array}{l}- \\
, 03634\end{array}$ & 04215 & $\begin{array}{l}- \\
, 123 \\
20\end{array}$ & $\begin{array}{l}, 050 \\
55\end{array}$ \\
\hline
\end{tabular}

Whether there is a meaningful difference between the average life span of the company or not according to the presence or absence of competition in the environment in which the enterprise is located can be found in the table above. (2-tailed) we can understand the value of. When the value of significance is less than 0.05 , there is a meaningful difference between the two groups. The significance value of our analysis is $0,529 \& \mathrm{gt}$; 0,05 , indicating that the average life span of the company did not differ significantly according to whether or not there was competition in the environment. The $\mathrm{H}_{16}$ hypothesis is rejected because the significance level of the average life span of the company is $p>0.05$ according to the presence of competition in the environment in which the operator is located. In other words, the average life span of the company does not differ significantly in terms of whether or not there is competition in the environment in which the operator is located

\section{The Impact of Operating Profitability on Company Average Life Span}

Dependent variable: Operational Profitability, Independent variable: Company average life span 
It is shown in Table 39 that the mean life time independent variable of the company is significant compared to the Anova test (Sig. $=0,000<0.05)$. It can be said that the profitability of the operation has made a significant contribution in explaining the average life span of the company.

Table 39. Anova Table for Company Average Life Span Independent Variable

\begin{tabular}{|l|l|l|l|l|l|l|}
\hline & Model & $\begin{array}{l}\text { Sum of } \\
\text { squares }\end{array}$ & df & Per Square & F & Sig. \\
\hline \multirow{2}{*}{1} & Regression & 3,661 & 1 & 3,661 & 7,865 &, $000^{\mathrm{b}}$ \\
\cline { 2 - 7 } & Waste & 67,969 & 99 &, 466 & & \\
\cline { 2 - 7 } & Total & 71,631 & 100 & & & \\
\hline
\end{tabular}

a. Dependent Variable: Operating Profitability

b. Fixed Value: Company life expectancy

Table 40. Regression Analysis Results for Company Average Life Span Independent Variable

\begin{tabular}{|l|l|l|l|l|l|l|}
\hline & Model & $\begin{array}{l}\text { Sum of } \\
\text { squares }\end{array}$ & df & Per Square & F & Sig. \\
\hline \multirow{2}{*}{1} & Fixed value & 3,438 &, 197 & & 17,428 &, 000 \\
\cline { 2 - 7 } & $\begin{array}{l}\text { Business } \\
\text { Profitability }\end{array}$ &, 159 &, 057 &, 226 & 2,804 &, 006 \\
\hline
\end{tabular}

a. Dependent Variable: Operating Profitability

b. Fixed Value: Company life expectancy

As seen in Table 40, the effect of the independent variable of the average life span of the company on customer loyalty is 0.159 . We can say that the average life span of the company has an effect on perception management because it affects the positive direction even though it has less effect than the other variables.

A positive relationship was found between the perception management of the textile firm managers and the average life span of the company. The $\mathrm{H}_{17}$ hypothesis has also been accepted.

\section{Results}

"Rarely" is the business profitability of textile managers who are continuing their activities in the province of Bursa and their participation in statements about average life span of the company.

The participation rate for the phrase "Failure to make any mistakes in their activities" is $100.0 \%$ if the business managers are in the frame of "Efforts to improve 
the image of the business" in the framework of business profitability opinions and in the opinion of the average life span of the company.

Textile management managers; There was no significant difference between gender, type of company, presence of competition in the business environment, and opinions about business profitability ( $p>.05)$. There is a significant difference $(p<.05)$ between their age, marital status, educational status, working time in the company, the number of employees in their companies and their views on operating profitability.

Textile management managers; There was no significant difference between the presence of competition in the environment where the sexes and businesses were located and the opinions about the average life span of the company ( $p>.05)$. There is a significant difference $(p<.05)$ between their age, marital status, educational status, working time in the company, type of company, number of employees in the company and their opinions about the average life span of the company.

The result is that the operating profit, which is considered as a dependent variable, reflects the variance of the company's life expectancy variable as $69.9 \%$, or in other words, the average life span of the company is $70 \%$ depending on operating profitability. When the profitability of operating profitability on the average life span of the company is $\mathrm{p}<0.05$, the operating profit of the company managers has an effect on the average life span of the company. The relationship between operating profitability and the average life span of the company was found to be significant at $\mathrm{p}<0.00$. According to this result, it can be said that the profitability of the company makes a meaningful contribution to the life expectancy of the company. 


\section{Disclosure statement}

No potential conflict of interest was reported by the author.

Email address: yusufk@gmail.com 


\section{References and notes:}

ATO. (2005). Press release and ATO reports, the average life expectancy of companies is 12 years. http://www.atonet.org.tr/Yeni/index.Php?P=331\&L=1 (Access date: April $14,2018)$

Akin, G. (2000). How long do things last? Journal of Organizational Change Management 13 (1), 30-31.

Aksoy, A., (1993), Operating Capital Management. Gazi Büro Bookshop, Ankara.

Aygün, M. (2012). The Impact of Working Capital on Company Performance: An Application on Turkish Manufacturing Sector. Ege Academic View / Ege Academic Rewiew, 12 (2), 215-223.

Barnard, C. (1938). The Functions of the Executive. Cambridge, Ma: Harvard University Press.

Brigham, E. F. \& amp; Houston, J. F. (2007). Fundamentals of Financial Management. USA: Thomson South - Western.

Capital. (2016). http://www.capital.com.tr/capital-dergi/history- CompaniesHaberdetay-2896 (Access date: April 14, 2018)

Clegg, S., Dunkerley, D. (2013). Organization, class and control. Routledge.

Çakır, A. (2000). Management of Receivables in an Inflationary Medium and an Application in Sivas. (Published Thesis). Cumhuriyet University Social Sciences Institute, Sivas.

De Geus, A. (1999). Living Company, (Translated by Ahmet Ünver). Rota Publications.

Hannan, M. T., Freeman, J. (1977). The population ecology of organizations. American journal of sociology, 929-964.

Katz, D., Kahn, R. L. (1966). The social psychology of organizations. New York: Wiley.

Kendall, J.E., Kendall, K.E. (1993). Metaphors and methodologies: living beyond the systems machine. MIS Quarterly, 17 (2), 149-171.

Tenant, M. (2000). Determination And Empirical Survey Of Working Capital 's Role In The Financial Failure With The Ratio Of Roles. (Published Thesis). Osmangazi University Institute of Social Sciences, Eskişehir.

Mammadova.R.(2019). Leadership effectiveness in Human Resource Management. Journal of Science and Innovative Technologies. DOI: 10.30546/26164418.10.2019.13. Azerbaijan, Baku.Number 10.pp.13-26

Meyer, M. W., Zucker, L. G. (1989). Permanently Failing Organizations. Newbury Park, Ca: Sage Publications.

Suárez, F., Utterback, J. M. (1995). Dominant designs and the survival of firms. Strategic Management Journal, 16 (6), 415-430.

Yildirim.M.( 2018). Effect of Psychological Pressure (mobbing) on Business life and Employee International Journal of Humanities and Social Development research. DOI: 10.30546/2523-4331.2018.2.2.39.Azerbaijan, Baku. Volume 2 .(2), 39-46 